\title{
Microscale wave breaking in stratified air-water pipe flow
}

\author{
P. Vollestad, ${ }^{1, \text { a) }}$ A.A. Ayati, ${ }^{1}$ and A. Jensen ${ }^{1}$ \\ 1. Department of Mathematics, University of Oslo
}

(Dated: 5 March 2019)

We perform an experimental analysis of two-phase stratified wavy pipe flow, with the aim to detect and quantify the effect of small scale wave breaking. Particle image velocimetry (PIV) is employed to analyze the velocity fields below individual waves, and a threshold for the vorticity on the leeward side of the crest is used to assess active wave breaking.

Keeping the liquid flow rate constant, we analyze five experimental cases with increasing gas flow rates. The cases span the flow map from when first interfacial waves are observed, to the "amplitude saturation" regime, where the rms interface elevation is independent of the gas flow rate. While some wave breaking events are observed also in the wave-growth regime, wave breaking is found to be much more frequent when the gas flow rate is increased into the amplitude saturation regime, and 35-40\% of the waves passing the measurement section are assessed to be in a state of active breaking in this regime.

A conditional averaging of the flow field is performed, and the turbulent dissipation rate below breaking and nonbreaking waves is estimated. The effect of microscale breaking is observed down to a depth of $10 \mathrm{~mm}$ below the water surface. Below the crest of microscale breaking waves the turbulent dissipation rate is increased by a factor 2.5 to 4 compared with non-breaking waves. This fraction increases with $U_{s g}$, implying that the breaking events become more energetic as the gas flow rate is increased.

\section{INTRODUCTION}

Wave breaking is characterized by fluid elements near the wave crest which achieve a velocity greater than the propagation speed of the wave, resulting in a reduction of the wave amplitude and increased turbulence levels in the liquid phase $^{1-3}$. Here we consider wave breaking at deep or intermediate depths, as discussed by Perlin, Choi, and Tian ${ }^{4}$, as opposed to depth-limited breaking, typically observed as water waves approach a beach ${ }^{5}$. The phenomenon is typically divided into categories related to the severity and visual characteristics of the breaking process. Babanin ${ }^{6}$ separates wave breaking into plunging, spilling and microbreaking. While wave breaking is generally associated with the more energetic forms, producing whitecaps on the water surface as air is entrained into the liquid phase, microbreaking or microscale wave breaking is a weak form of wave breaking without air entrainment ${ }^{1}$. For these small scale wave breaking events, surface tension prevents the jet formation and overturning of the wave crest which results in air entrainment for larger scale breaking events ${ }^{7,8}$. Recent numerical analysis by Deike, Popinet, and Melville ${ }^{9}$ demonstrates the effect of surface tension on the nature of the breaking process. As pointed out by Babanin ${ }^{6}$, while the external signature of microscale breaking waves are different to breaking waves that produce whitecapping, the fundamental physics is the same as for spilling breakers. Microscale breaking waves can also be referred to as small-wavelength spilling waves ${ }^{7,8}$. Due to the high frequency of microscale breaking on the ocean surface (compared with the more energetic forms of breaking producing whitecaps), it is assessed that microscale breaking may have a significant impact on the air-sea exchange in the ocean ${ }^{2,10}$. Recent field experiments indicate that microscale breaking waves and breaking events with very little

\footnotetext{
${ }^{a)}$ Corresponding author. E-mail: pettervo@math.uio.no
}

air-entrainment may contribute to $20-90 \%$ (depending on the wind speed and wave age) of the total dissipation by wavebreaking $^{11,12}$.

Microscale breaking is reported to occur for wind forced waves with wavelengths in the order of decimeters. Jessup, Zappa, and $\mathrm{Yeh}^{2}$ report the wavelength to be in the range of 0.1 to 1 meters, while Caulliez ${ }^{13}$ identified microscale wave breaking as the dominant dissipative mechanism for wind forced waves from 0.1 to $0.2 \mathrm{~m}$ wavelength. The visual characteristics of microscale breaking waves is a bore-like crest and parasitic capillary waves on the forward face of the wave ${ }^{2}$. As the breaking is initiated, a thin $(<2 \mathrm{~mm})$ spilling region is formed on the leeward face of the wave ${ }^{10}$. This region disrupts the parasitic capillaries closest to the crest, while the smaller capillary waves located upstream of the toe of the spilling region may prevail during the breaking event ${ }^{14}$. Recently, the kinematic breaking criterion, which states that breaking occurs when fluid particles at the wave crest moves faster than the representative wave velocity, has received significant support ${ }^{3,15,16}$. Investigations of the initiation of breaking is however outside the scope of the present work, as we focus on detecting actively breaking waves and evaluating the properties of the liquid flow below actively breaking and nonbreaking waves.

As microscale breaking waves do not cause air entrainment, they are more difficult to observe than breaking waves producing whitecaps. Different techniques have been applied to detect and quantify the effect of microscale breaking waves. Katsaros and Ataktürk ${ }^{17}$ used video recordings and a manual visual observation to detect and quantify microscale breaking waves in field conditions. Jessup, Zappa, and $\mathrm{Yeh}^{2}$ used infrared imagery and found that turbulence generated by the breaking process disrupts the thermal boundary layer of the microbreaking wave. According to Jessup, Zappa, and Yeh ${ }^{2}$, disruption of the thermal boundary layer and the infrared signature generated may serve as a practical means of defining microscale breaking waves. Siddiqui 
et al. ${ }^{18}$ used infrared imaging and subsurface particle image velocimetry (PIV) measurements and found that the disruption of the thermal boundary layer was well correlated with the presence of vortical structures and high levels of vorticity in the crest region of the wave. In a later work, Siddiqui and Loewen ${ }^{19}$ tested three different methods for detecting microscale breaking waves: wave steepness, infrared imagery and vorticity in the crest region evaluated by PIV. According to Siddiqui and Loewen ${ }^{19}$, PIV measurements of the vorticity field in the crest region was found to be the best predictor for detecting microscale breaking waves. In a later paper, Siddiqui and Loewen ${ }^{20}$ observed that below microscale breaking waves the turbulent dissipation rate increased by a factor two to three compared with non-breaking waves.

Peirson and Banner ${ }^{10}$ used PIV to evaluate the effect of microscale breaking waves. The spilling region was observed to remain compact and restricted to a limited portion of the overall waveform, downwind of the wave crest. Regions of intense shear were observed at the toe of the spilling region. In some PIV velocity fields vorticity was also observed downwind of the spilling region. This was attributed to the parasitic capillary waves riding in front of the wave. As demonstrated by Longuet-Higgins ${ }^{21}$, capillary waves located on the leeward side of short gravity waves generate intense vorticity in a thin layer close to the interface, but following the results from Peirson and Banner ${ }^{10}$ the impact of these capillary ripples are much smaller than the effect of microscale breaking.

In two-phase pipe flow, different flow regimes may be present, depending on the properties of the fluids involved and flow rate combinations applied. The stratified regime occurs when the gas and liquid velocities are relatively low, and below the threshold for transition to slug flow. As the gas velocity is increased, waves appear on the liquid interface. Within the stratified regime, several sub-regimes of wavy flow patterns exist, depending on the flow rate combinations ${ }^{22-25}$.

The main engineering aspects of the stratified flow regime is related to predicting flow parameters such as the pressure drop and liquid hold-up, and finding criteria for transition to slug flow. Due to the inherent complexities of the flow, simplified two-fluid models are the main tools applied. This approach depends on closure relations for the interfacial friction. A large number of proposed friction factor correlations can be found in the literature 26,27 .

Recently, PIV has been applied to study the stratified regime in two-phase pipe flow ${ }^{28-32}$. The main results from these studies are spatio-temporally and phase-averaged flow fields. While this has lead to an improved understanding of stratified gas-liquid pipe flow, the issue of microscale wave breaking has not yet been assessed in detail. Considering the significant impact of microscale wave breaking in the momentum budget at the atmosphere-ocean interface, it is assessed that small scale wave breaking may be an important mechanism also in the stratified wavy regime of two-phase pipe flow.

While some references to small scale wave breaking in twophase pipe flow are found in the literature, no thorough analysis of the phenomenon has been performed to the authors' knowledge. Coleman and Garimella ${ }^{24}$ used high speed cam- eras and observed wave breaking in the stratified wavy regime for sufficiently large pipe diameters. The severity of breaking was however not reported, nor was the point of breaking onset in the flow map specified. Ayati and Carneiro ${ }^{33}$ analyzed the statistics from interfacial wave probes in two-phase stratified flow, considering the same experimental setup and flow rate combinations closely matching the cases under investigation in the present work. By comparing the wave statistics with Gaussian statistics (linear wave theory), they observed a clear transition from a quasi-linear regime to a non-linear regime where linear wave theory considerably over predicts the maximum observed surface elevation $\eta$. The transition was linked to the onset of "amplitude saturation", reported previously by Ayati et al. ${ }^{34}$, where the rms surface elevation is found to be independent of the gas flow rate. Ayati and Carneiro ${ }^{33}$ mention microscale breaking as one of the possible mechanisms explaining the transition observed, but no further analysis was performed to assess whether the onset of microscale wave breaking corresponds with the observed transition.

In the present work we perform a PIV analysis of the liquid phase in stratified gas-liquid pipe flow. Keeping the liquid superficial velocity constant at $0.1 \mathrm{~m} / \mathrm{s}$ (the superficial velocity is the volumetric flow rate divided by the pipe cross-sectional area), the gas flow rate is varied and experimental cases covering the transition from the "wave growth" to the "amplitude saturation" regime observed by Ayati and Carneiro ${ }^{33}$ are considered. The goal is to identify where in the flow map microscale breaking waves first appear, and to quantify the effect of microscale wave breaking on the liquid phase flow field.

\section{EXPERIMENTAL SETUP}

The experiments were conducted at the hydrodynamics laboratory, University of Oslo. A horizontal, $31 \mathrm{~m}$ long transparent acrylic pipe with an internal diameter $\mathrm{D}=100 \mathrm{~mm}$ is used. The fluids are air and water at atmospheric pressure. An overview of the experimental setup is presented in figure 1. Water is supplied at the inlet through a $50 \mathrm{~mm}$ diameter pipe. The water mass flow rate was controlled by a frequency regulated pump, and measured using an Endress Hauser Promass coriolis flow meter. The air flow was supplied by a frequency regulated fan, and measured by an Emerson MicroMotion Coriolis flow meter. The water and air flow meters have an accuracy of $\pm 0.2 \%$ and $\pm 0.05 \%$ of maximum measured value, respectively. Honeycomb flow straighteners were placed at the inlet of the pipe section to dampen turbulent fluctuations introduced at the inlet. The differential pressure in the air phase was measured over a 12.4 meter section, using a smar LD301 differential pressure gauge $( \pm 0.2 \%$ of max. range in accuracy).

A PIV section was placed approximately $260 \mathrm{D}$ downstream of the inlet. An optical correction box was placed covering the PIV section. The box was half filled with isopar which ensured that the optical distortion of the liquid phase PIV camera was reduced. A three-camera system was applied in the study. The camera specifications and their function in the experimental setup is summarized below. The placement 


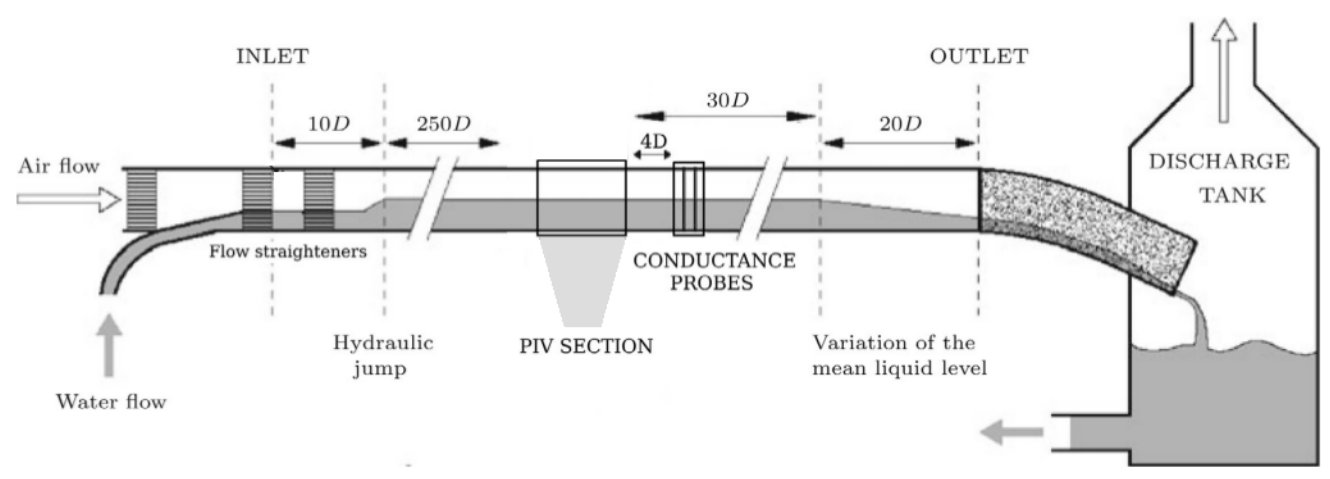

FIG. 1: Schematic view of experimental setup.

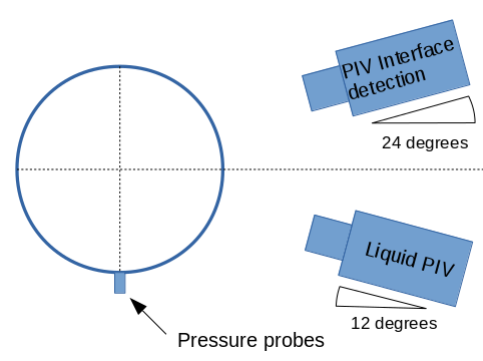

a)

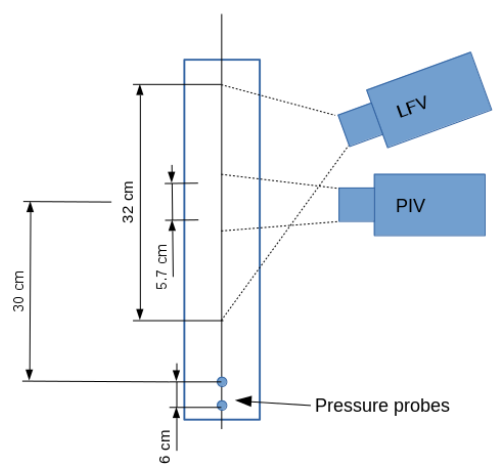

b)

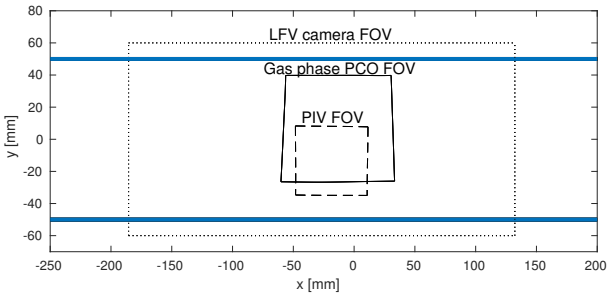

c)

FIG. 2: Schematic view of PIV setup. a) Looking downstream. The LFV (large field of view) camera is located at the same height as the gas phase PCO camera. b) Seen from above. The liquid phase PIV camera is located below the gas phase PCO camera. c) Field of view along the centerline of the pipe for the three cameras.

of the cameras and their field of view (FOV) is illustrated in figure 2 .

- Liquid phase PIV camera. A 14 bit PCO.4000 camera with $4008 \times 2672$ pixels, oriented upwards at an angle of $12^{\circ}$. The camera was fitted with an AF-S Teleconverter and a $100 \mathrm{~mm}$ lens. This enabled a relatively small FOV of $57 \mathrm{~mm}$ in the horizontal direction.

- PIV interface detection camera. Same type of camera as the liquid phase PIV camera, but fitted without the AF-S Teleconverter. Directed downward at an angle of $24^{\circ}$, focused on the interface. A high downward looking angle was necessary as the crescent shape of the waves meant that the pipe wall was repeatedly wetted by the waves well above the mean liquid level. FOV of approximately $100 \mathrm{~mm}$ in the horizontal direction.

- Large-field of view (LFV) camera. A Nikon D7200 (4800x3200 pixels), equipped with a $28 \mathrm{~mm}$ lens was directed at an angle of approximately $30^{\circ}$ downwards, looking upstream at an angle of approximately $31^{\circ}$. FOV of approximately $320 \mathrm{~mm}$, which is longer than the dominant wavelengths of all experimental cases in the study. Images were used to identify the large scale properties (such as the wavelength and height) of the waves.

Prior to the experiments, a coordinate system was inserted into the pipe centerline, and cubic coordinate transforms (from pixel to world coordinates) were created for each of the three cameras. The coordinate system is identical to the one used by Ayati et al. ${ }^{34}$, but has been extended in the horizontal direction due to the larger FOV of the LFV camera.

A vertical plane in the pipe center was illuminated from below by a laser sheet, generated by a $147 \mathrm{~mJ}$ double pulsed ND:YAG laser. The head of the laser optics was placed 45 $\mathrm{cm}$ below the optical correction box. This enabled the laser light sheet to illuminate not only the FOV of the PIV, but the full FOV of the LFV camera. The two laser pulses were triggered with a $\Delta t$ of $300 \mu \mathrm{s}$. The PCO cameras acquired double images (one image per laser pulse) used for PIV calculations, while the LFV camera was set to trigger before the first camera pulse, and has an exposure time which extends over the time period of both laser pulses. Hence, the LFV image is double exposed with a $\Delta t$ of $300 \mu s$. During this time delay the waves in the highest gas flow rate case will move approximately $0.25 \mathrm{~mm}$, which is a negligible distance compared with the overall wave shape.

The liquid phase was seeded with $20 \mu \mathrm{m}$ polyamide 
spheres. PIV was performed using a cascade of crosscorrelation passes, with a final subwindow size of $32 \times 32$ pixels, and $50 \%$ overlap. This translates to a spatial resolution of approximately $0.24 \times 0.24 \mathrm{~mm}$. Spurious vectors were detected by standard methods based on a local median filtering ${ }^{35}$. Typically, 2-3\% of the vectors were found to be spurious, and replaced by cubic interpolation. Note that for microscale breaking waves, light reflections were detected at the interface of the spilling region. In order to avoid that these bright regions dominated the PIV results, these high intensity regions were masked from the raw images prior to the PIV analysis. Rhodamine B was added to the water at a concentration of approximately $0.1 \mathrm{mg} / \mathrm{L}$ to enhance the contrast between the liquid and gas phase, enabling efficient interface detection from the two gas-phase cameras.

A conductance wave gauge was placed $4 D$ downstream of the PIV section. This consists of two double-wire probes of $0.3 \mathrm{~mm}$ diameter separated by $4 \mathrm{~mm}$. The two probes were placed in the center of the pipe with a distance $6 \mathrm{~cm}$ in the streamwise direction. The interface elevation was measured with a relatively high temporal resolution of $500 \mathrm{~Hz}$. For more details about the wave-gauges the reader is referred to Ayati et al. ${ }^{34}$.

The data transfer rate between the cameras and the computer limited the frequency of data acquisition, and it was found necessary to reduce the PIV acquisition rate to maximum $0.2 \mathrm{~Hz}$ for the data transfer to be successful over long measurement series (i.e. a new set of double images could be acquired every 5 seconds). To overcome the limitation of the low PIV acquisition rate, and to ensure that the PIV system acquired images of value to the experimental investigation, a system to trigger the PIV acquisition was designed. Two pressure probes (Kulite XTL-190) mounted flush to the pipe bottom (position indicated in figure $2 \mathrm{a}-\mathrm{b}$ ) detected as waves approached the PIV section, and triggered the PIV system, ensuring that the leeward side of the waves (where we expect to see the strongest indications of wave breaking) were regularly present within the PIV FOV. As we are interesting in detecting breaking waves, the triggering system was set up to detect the relatively larger waves in the system, where breaking is assessed to be more probable. Details of the triggering system is presented in "Appendix A: Trigging of PIV system".

\section{METHODOLOGY}

\section{A. Description of experimental cases}

Five experimental cases, where the liquid superficial velocity is kept constant at $U_{s l}=0.1 \mathrm{~m} / \mathrm{s}$, while the gas superficial velocity is increased from 1.5 to $2.4 \mathrm{~m} / \mathrm{s}$ are analyzed. The main characteristics of the experimental cases are presented in table I. $U_{s f}, U_{b f}$ is the superficial and bulk velocities, where $f=g, l$ refers to the gas or liquid phase respectively. $U_{b f}=U_{s f} A / A_{f}$, where $A$ is the total cross-sectional area of the pipe, while $A_{f}$ is the area occupied by phase $f . R e_{D f}$ is the Reynolds number based on the hydraulic diameter. The number of PIV images acquired for each case is presented in the right column of table I. As we will analyze breaking and non-breaking waves separately, a larger number of velocity fields were acquired in the cases where wave breaking was present.

Additional information including the mean water level, rms of the interface elevation fluctuations $\left(\eta_{r m s}\right)$, characteristic wave speed $C_{p}$ (calculated by cross-correlation of the conductance wave probes), and dominant wavelength $\lambda_{p}$ (evaluated based on the spectral peak frequency and characteristic wave speed, $\left.\lambda_{p}=C_{p} / f_{p}\right)$, as well as mean pressure drop $(\Delta p / \Delta x)$ is presented in graphical form in figure 3 , while the normalized wave spectra for all five cases under investigation is presented in figure 4 . Note that in figure 3 additional experimental cases with flow rates lower and higher than the cases investigated by PIV have been included, to better visualize the trends in the flow map. Here the PIV cases under investigation are marked by crosses.

Different regimes exist within two-phase stratified pipe flow, depending on the flow rate combinations applied, and the properties of the fluids. Andritsos and Hanratty ${ }^{26}$ and Tzotzi and Andritsos ${ }^{22}$ discussed four sub-regimes within stratified flow:

(i) Smooth regime (no waves)

(ii) Two-dimensional (2D) wave regime. Interface is covered by small amplitude, relatively regular disturbances.

(iii) Large amplitude (Kelvin-Helmoltz) waves regime.

(iv) Atomization regime.

Applying the expressions proposed by Tzotzi and Andrit$\operatorname{sos}^{22}$ for predicting flow regime transitions, the $2 \mathrm{D}$ wave regime is predicted to be present for $1.1 \mathrm{~m} / \mathrm{s}<U_{s g}<4.1 \mathrm{~m} / \mathrm{s}$ in our experimental setup. This is in good agreement with visual observations of the flow field, although in the experimental setup we first observed waves when $U_{s g}$ exceeded 1.3 $\mathrm{m} / \mathrm{s}$. Variations in pipe diameter, inlet conditions and pipe materials is however known to impact on the transition criteria. The three-dimensionality of the wavefield has previously been investigated by Strand ${ }^{25}$. Using the same experimental setup and liquid flow rate he found that the spanwise crosscorrelation was close to unity for $U_{s g}<4 \mathrm{~m} / \mathrm{s}$, indicating that the waves are well described as 2D waves. For higher gas flow rates Strand ${ }^{25}$ found that the spanwise cross-correlation was drastically reduced, indicating the transition to the large amplitude wave regime. We conclude that all experimental cases considered in this work are within the $2 \mathrm{D}$ wave regime.

Figure 3 illustrates the dominant trends in the flow field as $U_{s g}$ is increased. Starting with no waves $\left(\eta_{r m s} \approx 0.2 \mathrm{~mm}\right.$ identified as electrical noise by Ayati et al. ${ }^{34}$ ) at $U_{s g}=1.30 \mathrm{~m} / \mathrm{s}$, the wave field passes through a regime of amplitude growth, until the "amplitude saturation" regime is observed at $U_{s g}=$ $2.10 \mathrm{~m} / \mathrm{s}$. Hence the five experimental cases under investigation cover the range from amplitude growth to saturation. Figure 3 b-d) shows that while also the dominant wavelength stabilizes as $U_{s g}$ is increased, the mean water level is reduced (due to increased interfacial friction), while the wave speed and pressure drop is increased. The wave speed is observed 
TABLE I: Experimental cases under investigation. $U_{s l}=0.1$ $\mathrm{m} / \mathrm{s}$ for all cases. $N_{P I V}$ is the number of PIV velocity fields acquired per experimental case.

\begin{tabular}{|c|c|c|c|c|c|}
\hline $\begin{array}{c}U_{s g} \\
{[\mathrm{~m} / \mathrm{s}]}\end{array}$ & $\begin{array}{c}U_{b l} \\
{[\mathrm{~m} / \mathrm{s}]}\end{array}$ & $\begin{array}{c}U_{b g} \\
{[\mathrm{~m} / \mathrm{s}]}\end{array}$ & $\begin{array}{c}\operatorname{Re}_{D g} \\
{[-]}\end{array}$ & $\begin{array}{c}\operatorname{Re}_{D l} \\
{[-]}\end{array}$ & $N_{P I V}$ \\
\hline $\mathbf{1 . 5 0}$ & 0.26 & 2.44 & 11400 & 23800 & 500 \\
\hline $\mathbf{1 . 7 0}$ & 0.27 & 2.75 & 13000 & 23100 & 500 \\
\hline $\mathbf{1 . 8 0}$ & 0.27 & 2.95 & 13700 & 24200 & 1000 \\
\hline $\mathbf{2 . 1 0}$ & 0.30 & 3.22 & 15600 & 25300 & 1000 \\
\hline $\mathbf{2 . 4 0}$ & 0.30 & 3.67 & 17800 & 25200 & 1000 \\
\hline
\end{tabular}

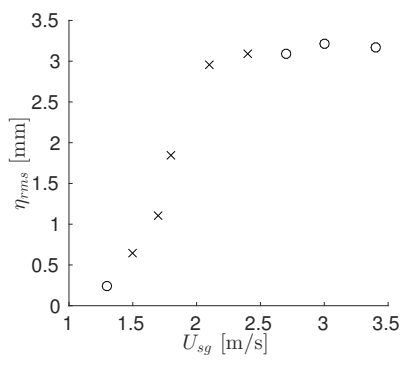

a) $\eta_{r m s}$

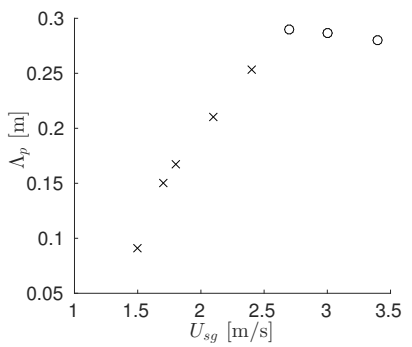

c) $\Lambda_{p}$

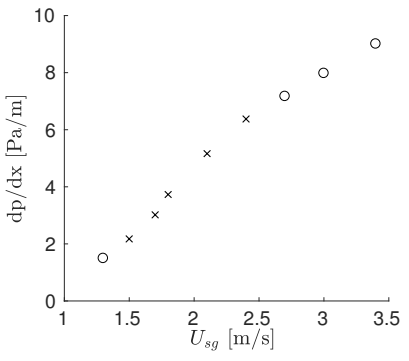

e) $d p / d x$

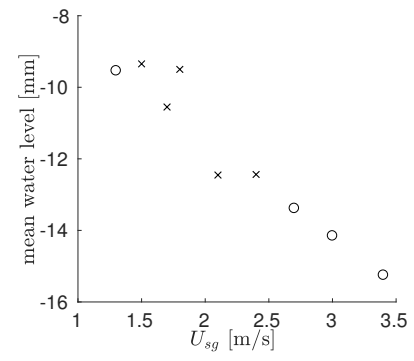

b) mean water level

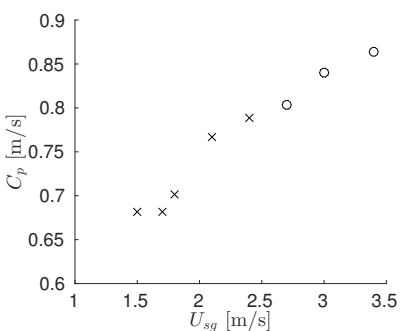

d) $C_{p}$

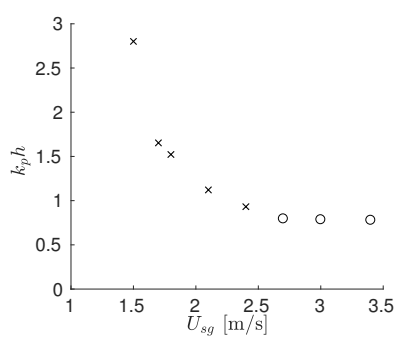

f) $k_{p} h$
FIG. 3: Data for the experimental cases. $\mathrm{x}$ : cases analyzed by PIV. Additional data points (o) included to visualize the trends in the flow map. a) $\eta_{r m s}$ is the rms interface elevation. b) mean water level (relative to the pipe centerline). c) Peak wavelength ( $\lambda_{p}$, evaluated from spectra). d) Characteristic wave speed $C_{p}$. e) $d p / d x$, average pressure drop over 12.4 meter section of the pipe. f) $k_{p} h$ ( $h$ is mean water level in pipe center).

to increase despite the fact that the geometrical features of the waves (amplitude and wavelength) and $k_{p} h$ are relatively constant above $U_{s g} 2.70$. This is related to the reduced water level and the increased bulk water velocity (as the volumetric liquid flow rate is constant for all cases).

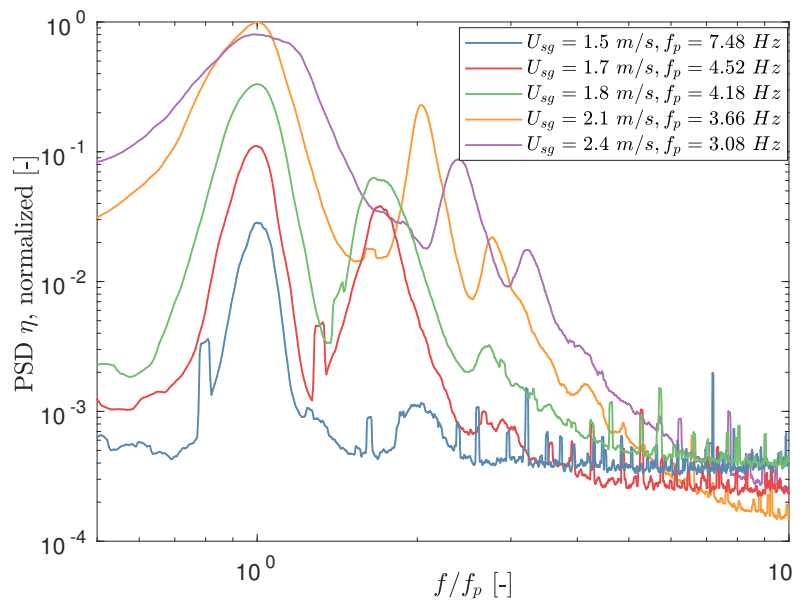

FIG. 4: Normalized waveprobe frequency spectra for the five cases under investigation. $y$-axis normalized by the maximum spectral value, $\mathrm{X}$-axis normalized by peak frequency $f_{p}$ for each experimental case (included in figure legend).

\section{B. Interface and wave phase detection}

The liquid interface was first found from the LFV images, using a thresholding technique. Transforming this interface to the gas phase PCO images illustrated that while the interface detected by the LFV camera provided adequate accuracy to describe the large scale properties of the waves, improved accuracy of the interface in the PIV section could be obtained by the gas phase PCO images. Hence, and a region 100 pixels above/below the interface found by the LFV camera was scanned along the PCO images, using a combination of sobel edge detection and a threshold value of the light intensity to detect the interface. The interface was then converted to the liquid phase PIV images, and the region above the interface was masked and omitted from the PIV analysis. By analyzing original PIV images and the interfaces obtained, the accuracy of the interface detection is estimated to be $0.25 \mathrm{~mm}$, equivalent to the PIV resolution.

The wave phase as a function of horizontal coordinate was calculated based on the LFV interfaces by a zero-crossing procedure. Crests, troughs and zero crossings were assigned a phase of $0^{\circ}, 180^{\circ}$ and $\pm 90^{\circ}$ respectively, and the phases in between were linearly distributed. The process is illustrated in figure 5. This allows for statistics of the flow field located at the same relative location of the wave to be evaluated, and is a crucial step in the conditional phase-averaging procedure described in section III C, and the classification into breaking and non-breaking waves presented in section IV B.

\section{Phase-averaging procedure}

A conditional averaging of all scalar fields (horizontal velocity, vertical velocity, vorticity, etc.) is performed on a wave-following coordinate system. For each velocity field a 


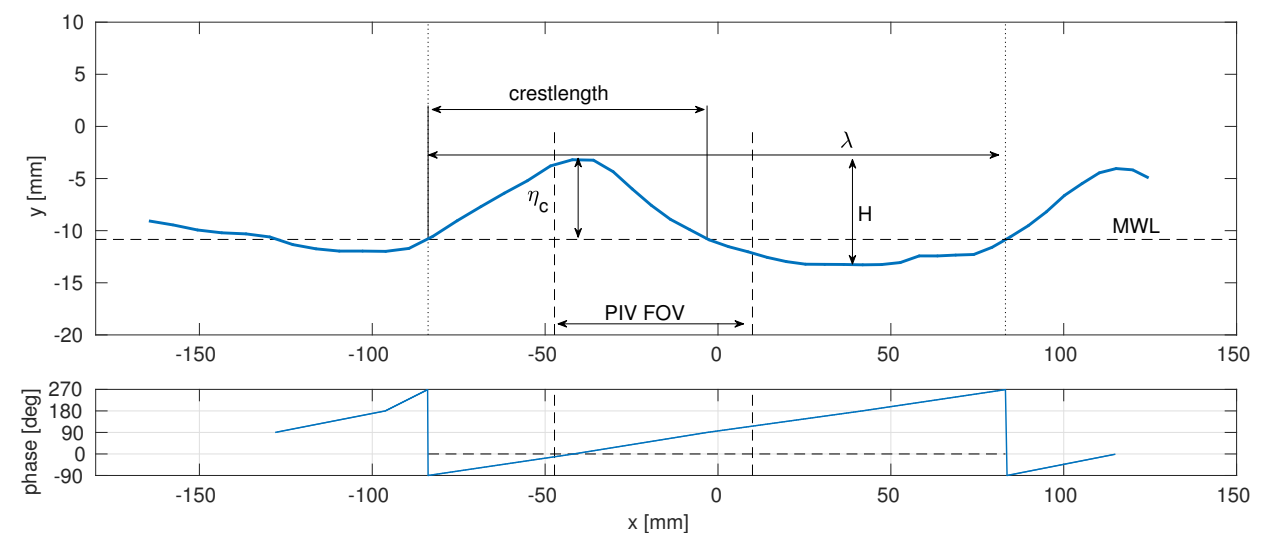

FIG. 5: Example plot illustrating the interface detection (top) and wave phase determination (bottom). Thick blue line: Interface detected from LFV camera. PIV FOV and wave properties including $\eta_{c}, \lambda$ and the crestlength indicated.

coordinate transformation from $(x, y)$ to $(\theta, \zeta)$ is performed.

The wave-following coordinate system used is similar to the coordinate system applied by Birvalski et al. ${ }^{30}$ and Siddiqui and Loewen ${ }^{20}$. The wave phase $\theta$ determined by the LFV camera (ref. section III B) is used together with a relative height between the interface and the lower part of the PIV velocity field (located at $\mathrm{y}=-30 \mathrm{~mm}$ ) to create a wave-following coordinate system (illustrated in figure 6). The height between the interface and $\mathrm{y}=-30 \mathrm{~mm}$ is divided into 100 equally spaced points and the scalar fields are sampled at each of these 100 nondimensional $\zeta$-coordinates and at each wave phase $(\Delta \theta=1)$ observed in the FOV.

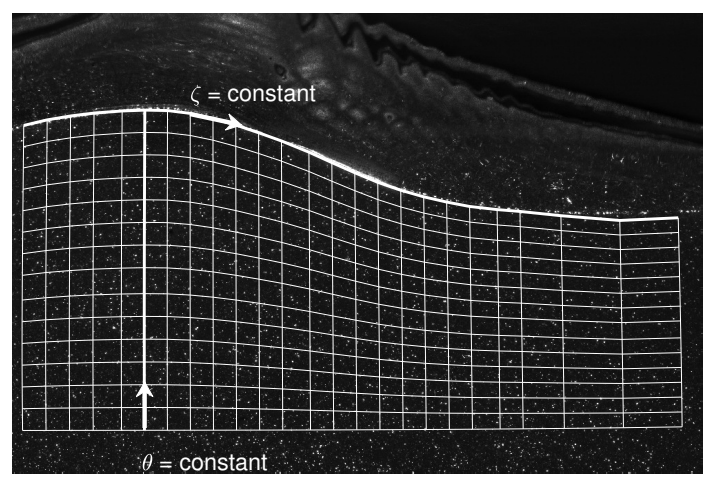

FIG. 6: Illustration of the wave-following coordinate system applied, overlaid original PIV image. Vertical lines are lines of constant $\theta$, near horizontal/wave-following lines are lines of constant $\zeta$.

Once sampled, the identified velocity fields are ensambleaveraged (in the $(\theta, \zeta)$ space). This ensures that the ensamble of velocity fields are averaged at constant wave phase and at a constant relative depth between the interface and the lower part of the PIV FOV. The phase-averaged interface elevation is evaluated simultaneously. When presenting the phaseaveraged properties, the wave field is transformed from $(\theta, \zeta)$ to $(\theta, y)$ by applying the mean interface elevation.

\section{Estimation of turbulent dissipation rate}

When sampling the velocity field in a wave-following coordinate system a three-component Reynolds decomposition of the velocity field is typically applied ${ }^{30,36,37}$. For a given variable $q$ in the flow field, which varies in time and space, the variable is decomposed into a phase independent mean $\bar{q}(\zeta)$, a wave induced field $\tilde{q}(\theta, \zeta)$ and a fluctuating component $q^{\prime}(\theta, \zeta, t)$ :

$$
q(x, y, t)=\bar{q}(\zeta)+\tilde{q}(\theta, \zeta)+q^{\prime}(\theta, \zeta, t)
$$

The phase-averaged component $\langle q\rangle$ is then

$$
\langle q\rangle(\theta, \zeta)=\bar{q}(\zeta)+\tilde{q}(\theta, \zeta)
$$

The above method is not able to fully decouple the waveinduced motions and the turbulence in a dispersive wave system $^{30}$. As the system consists of a (relatively) broad banded spectrum of wave components, a significant amount of the wave-coherent velocity fluctuations will be included in the fluctuating velocity component. These errors will magnify when quantities such as the turbulent kinetic energy is calculated from the three-component Reynolds decomposition.

Siddiqui and Loewen ${ }^{38}$ introduced a spatial filtering technique to estimate the turbulent velocity fluctuations beneath microscale breaking waves. When the mean velocity $\bar{u}$ is subtracted from the instantaneous velocity field, we are left with the wave-coherent and turbulent fluctuations. This is referred to as $u_{w t}$. Wave-coherent fluctuations $u_{w}$ are estimated by applying a spatial low-pass filter to the $u_{w t}$ field, where the cutoff wavenumber is chosen to be lower than the wavenumber associated with the largest and most energetic turbulent structures. The rationale for this method is that the wave-coherent fluctuations occur over a larger spatial scale than the turbulent fluctuations generated by breaking. The turbulent fluctuations are then estimated as $u_{t}=u_{w t}-u_{w}$.

It is observed that beneath microscale breaking waves a turbulent region with high levels of vorticity is observed to extend typically $6 \mathrm{~mm}$ below the interface (see section IV A). 
Based on this observation, it is assessed that the most energetic turbulent structures generated by the breaking waves are in the range of $6 \mathrm{~mm}$, and a spatial low-pass filter of $6 \times 6 \mathrm{~mm}$ (top-hat) is applied. Note that changing the filter size in the range of $4 \times 4$ to $15 \times 15 \mathrm{~mm}$ was tested and found to have only a small quantitative effect of the results. Figure 7 illustrates the effect of the low-pass filter, and the evaluation of $u_{t}$.

The low-pass decomposition technique is not fully able to decouple the turbulent fluctuations from the wave-induced fluctuations ${ }^{38}$. As the wave-induced fluctuations can be more than an order of magnitude higher than the turbulent fluctuations, including even small fractions of wave-coherent fluctuations into the turbulent fluctuations can lead to significant overestimation of quantities such as the turbulent kinetic energy. Hence, this method should not be applied to calculate the turbulent kinetic energy or Reynolds stresses.

The dissipation rate of turbulent kinetic energy can be estimated from a 2D velocity field using the following relation $^{38,39}$ :

$$
\begin{gathered}
\varepsilon=3 v\left(\overline{\left(\frac{\partial u^{\prime}}{\partial x}\right)^{2}}+\overline{\left(\frac{\partial v^{\prime}}{\partial y}\right)^{2}}+\overline{\left(\frac{\partial u^{\prime}}{\partial y}\right)^{2}}+\overline{\left(\frac{\partial v^{\prime}}{\partial x}\right)^{2}}+\right. \\
\left.2 \overline{\left(\frac{\partial u^{\prime}}{\partial y} \frac{\partial v^{\prime}}{\partial x}\right)}+\frac{2}{3} \overline{\left(\frac{\partial u^{\prime}}{\partial x} \frac{\partial v^{\prime}}{\partial y}\right)}\right)
\end{gathered}
$$

where the overbar indicates time or ensamble average. As can be seen from equation $3, \varepsilon$ is only dependent on the spatial derivatives of the fluctuations, not on the magnitude of the fluctuations. In this work the spatial derivatives of the fluctuations are evaluated by a $5 \times 5$ finite difference filter, considering the $50 \%$ overlap used in the PIV processing, and to reduce the influence of small errors in the PIV. Siddiqui and Loewen ${ }^{38}$ argue that as the spatial derivatives of $u_{t}$ are higher than the spatial derivatives of $u_{w}$, the method outlined above can be applied to give a reasonable estimate for $\varepsilon$, as the inclusion of wave-coherent fluctuations into $u_{t}$ will not significantly alter the spatial derivatives of $u_{t}$. In the experiments by Siddiqui and Loewen ${ }^{38}$, the turbulent velocity gradients were typically 2.5 times larger than the wave-induced velocity gradients in the crest region. Similar results were obtained in this study.

In order for the method to be accurate, PIV needs sufficient spatial resolution to resolve the shear responsible for the dissipation. The dissipation of turbulent kinetic energy occurs at the smallest scales of the flow, bounded by the Kolmogorov microscale $\eta_{v}$. As discussed by Siddiqui and Loewen ${ }^{38}$, it is not necessary to fully resolve the Kolmogorov scale for the dissipation rates to be estimated with reasonable accuracy, as most of the dissipation occurs at scales larger than $\eta_{v}$. The Kolmogorov length scale can be evaluated as $\eta_{v}=\left(v^{3} / \varepsilon\right)^{0.25}$. As $\varepsilon$ is the magnitude we are interested in, calculating $\eta_{v}$ a priori is not possible, and applying the obtained dissipation rates to estimate $\eta_{v}$ implies a circular argument. Here we use a simplified approach to estimate $\eta_{v}$ a priori. Using the triple decomposition described in equation 1 , we estimate the turbulent kinetic energy in the flow field. For the highest gas flow rate case the maximum turbulent kinetic energy evaluated directly below the wave crest was estimated to be $3.75 * 10^{-3}$ $m^{2} / s^{2}$. This implies a characteristic turbulent velocity $u^{\prime}$ of $0.05 \mathrm{~m} / \mathrm{s}$. Note that this is assessed to be a high estimate for the turbulence in the region, as wave-coherent velocities which were not accurately filtered by the three-component Reynolds decomposition will impact on the estimated turbulent kinetic energy. The region of high turbulence levels below the interface of microbreaking waves typically extended $6 \mathrm{~mm}$ below the interface. With this velocity and length scale we estimate a characteristic eddy turnover time for the most energetic eddies as $\tau_{\text {turnover }}=l / u^{\prime}$. If it is further assumed that all the turbulent kinetic energy is dissipated within the time period $\tau_{\text {turnover }}$ (assessed to be a conservative assumption), $\varepsilon \approx k / \tau_{\text {turnover }}$. Applying these assumptions we can provide an estimate of $\varepsilon$ directly beneath the crest of $0.03 \mathrm{~m}^{2} / \mathrm{s}^{3}$ (this estimate is seen to be in fair agreement with results obtained in section IV C 2), which results in an estimate of $\eta_{v}$ of $73 \mu \mathrm{m}$. The smallest structures which can be detected by the PIV are $\approx 480 \mu m$ (twice the PIV resolution), which gives $k \eta_{v} \approx 1$. From the normalized energy and dissipation rate spectra presented by Tennekes and Lumley ${ }^{40}$, it can be seen that almost all the dissipation $\left(>90 \%\right.$ ) occurs for $k \eta_{v}<1$, indicating that the resolution of the PIV is sufficient for giving reasonable estimates for $\varepsilon$ beneath the crest.

Using the estimate for $\varepsilon$, we can estimate the kolmogorov timescale as $\tau_{k}=(v / \varepsilon)^{0.5}$, and find that the $\Delta t$ in the PIV acquisition is more than an order of magnitude lower than $\tau_{k}$. Hence we conclude that the spatial resolution, not the temporal resolution used to compute individual PIV velocity fields is the limiting factor in determining $\varepsilon$.

In the highly turbulent spilling region the turbulence levels are observed to be an order of magnitude higher than directly below the crest. Here the high turbulence region is observed to be very thin, and it is assessed that the Kolmogorov length scale is smaller than what we are able to resolve with the current PIV method, making the estimated dissipation rate in this region unreliable.

\section{RESULTS}

\section{A. Instantaneous Velocity fields}

In this section a few example plots of the velocity and vorticity fields of individual PIV results are presented.

Figure 8 illustrates a wave which is not in an active state of breaking. In figure 8 a) capillary ripples are observed on the leeward face of the wave, extending almost to the crest of the wave. A vector plot of the liquid phase (seen in a frame of reference moving with the wave speed) is presented in figure $8 \mathrm{~b}$ ). Here it is seen that the wave speed is higher than the liquid velocity vectors. The vorticity contour plot presented in figure $8 \mathrm{c}$ ) shows that the vorticity is relatively constant along the wave profile. However, somewhat higher vorticity levels $\left(\approx-100 s^{-1}\right)$ are observed just beneath the wave crest. This is assessed to be caused by the higher wind forcing exerted on the interface in this region. As mentioned in the intro- 


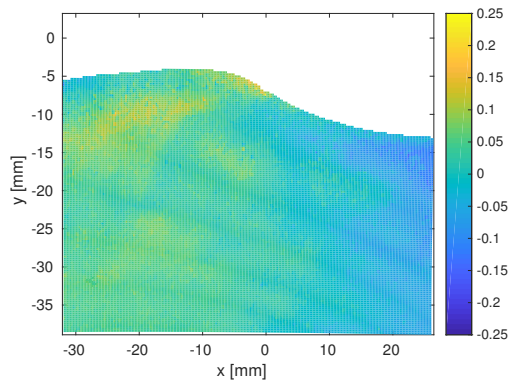

a) $u_{w t}$

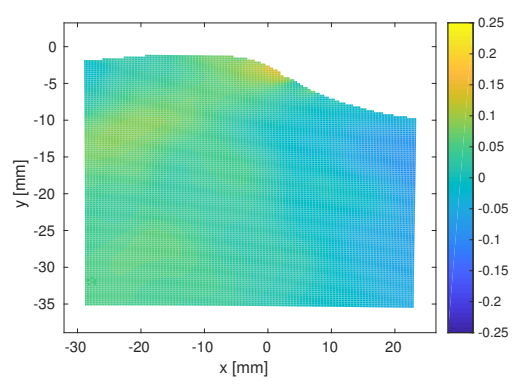

b) $u_{w}$

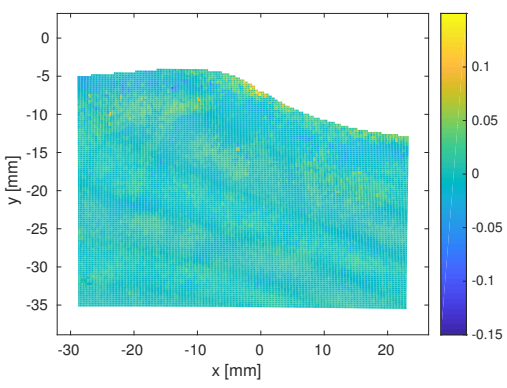

c) $u_{t}$

FIG. 7: Illustration of the spatial high-pass decomposition of the (horizontal) turbulent and wave-coherent velocity fluctuations. Colorbar in $\mathrm{m} / \mathrm{s}$.

duction, a bore-like crest and capillary ripples as observed in figure 8 a) and d) are characteristic of microscale breaking waves. However, following the discussion by Qiao and Dun$\mathrm{can}^{41}$ on the time development of small scale breaking waves, increased levels of vorticity and energy dissipation is first observed when the leading edge of the bulge of the crest starts to move downwards, creating a highly turbulent flow where water particles are observed to move at a velocity exceeding the wave speed. While the formation of the bulge and the parasitic capillaries can be associated with wave breaking, we will only refer to the wave as breaking when increased levels of vorticity are observed beneath the leeward side of the wave, i.e. when the bulge has started to move down the wave resulting in a highly turbulent flow. The wave presented in figure 8 can be considered as incipient breaking. Note that the majority of the non-breaking waves do not exhibit the distinct bulge and capillary ripples presented in figure 8 , as the non-breaking waves are typically much smoother.

It can be noted that no significant increase in vorticity is observed beneath the parasitic capillary waves observed in figure 8 a) and d). Following Longuet-Higgins ${ }^{21}$, very high levels of vorticity is expected to be generated beneath small capillary waves. However, as discussed by Duncan ${ }^{8}$, the layer of increased vorticity directly generated by parasitic capillary waves will be very small, significantly smaller than what we are able to resolve using PIV. Our observations follow the results by Qiao and Duncan ${ }^{41}$ and Peirson and Banner ${ }^{10}$, and indicate that diffusion of vorticity from parasitic capillary waves into the interior of the wave field is a weak effect relative to the onset of wave breaking.

Figure 9 represents a wave which is breaking. The vector plots presented in figure $9 \mathrm{~b}$ ) shows that the spilling region is highly turbulent, and the maximum horizontal velocities exceed the wave propagation speed. The capillary ripples which in figure 8 were observed to extend all the way to the crest are now confined to a small region below the spilling region. The vorticity contour plot presented in figure $9 \mathrm{c}$ ) shows that high levels of vorticity (peak vorticity of $\approx-1000 s^{-1}$ ) are produced in the spilling region, and a turbulent wake of high vorticity is observed below the crest.

The PIV system will detect waves at different stages of the wave breaking process. From visual inspection of the flow field (also using high-speed video), and looking through many images similar to the ones presented in this section, it is assessed that the velocity field presented in figure 9 represents a typical wave in an intermediate stage of the breaking process. Here the shape of the wave is still relatively similar to the nonbreaking wave presented in figure 8 , and the region of highest vorticity is observed in a relatively thin layer (approximately $3 \mathrm{~mm}$ ) along the leeward side. Towards the windward side of the wave a marked reduction of the vorticity is observed.

Waves assessed to be in a later stage of the breaking process were frequently observed in the experimental data. These were characterized by a more irregular shape of the crest, and high levels of vorticity was observed throughout the crest, down to a depth of up to $10 \mathrm{~mm}$ beneath the free surface. Here the high vorticity region was observed to extend a significant distance in the upstream direction, possibly impacting the upstream wave as this overtakes the turbulent eddies left in the wake of the breaking wave (limitations in the PIV FOV meant that this effect could not be observed directly). 


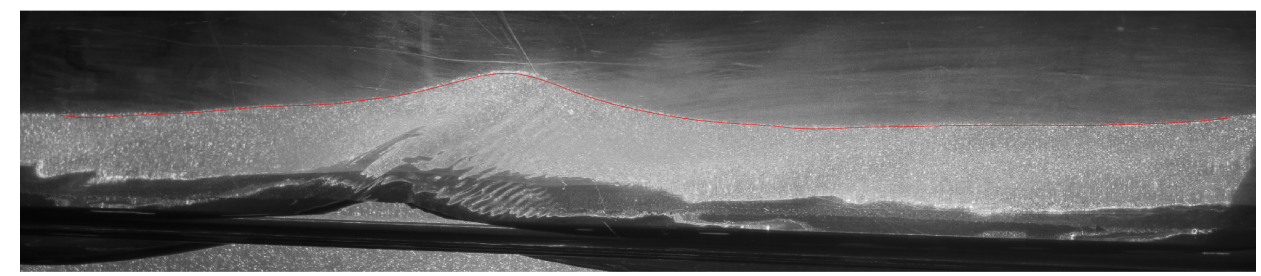

a)

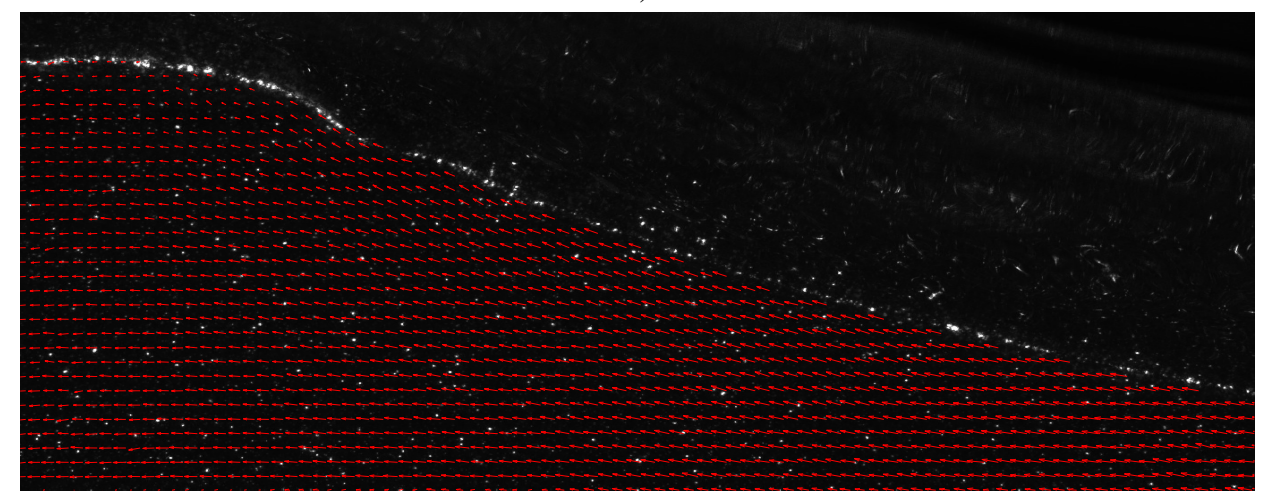

b)

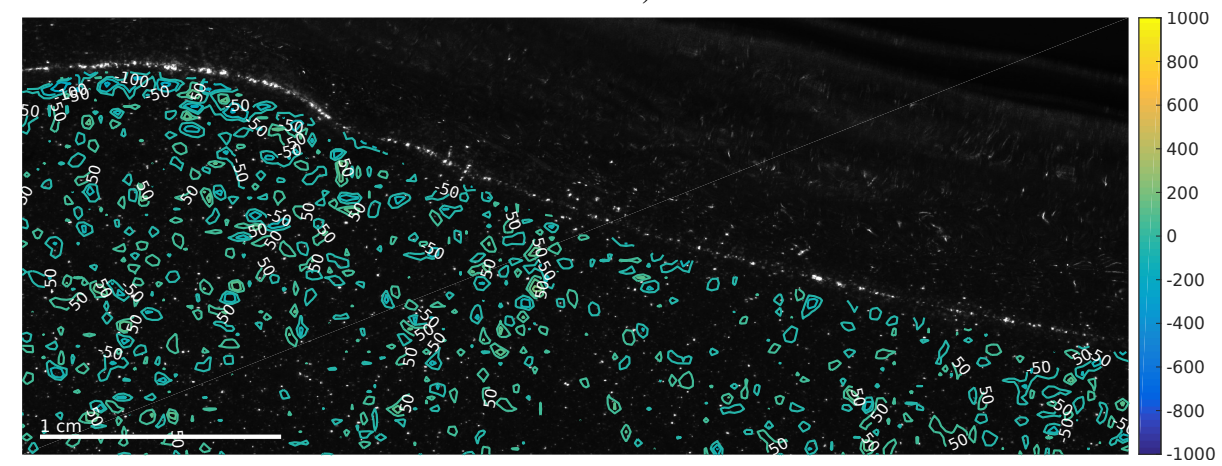

c)

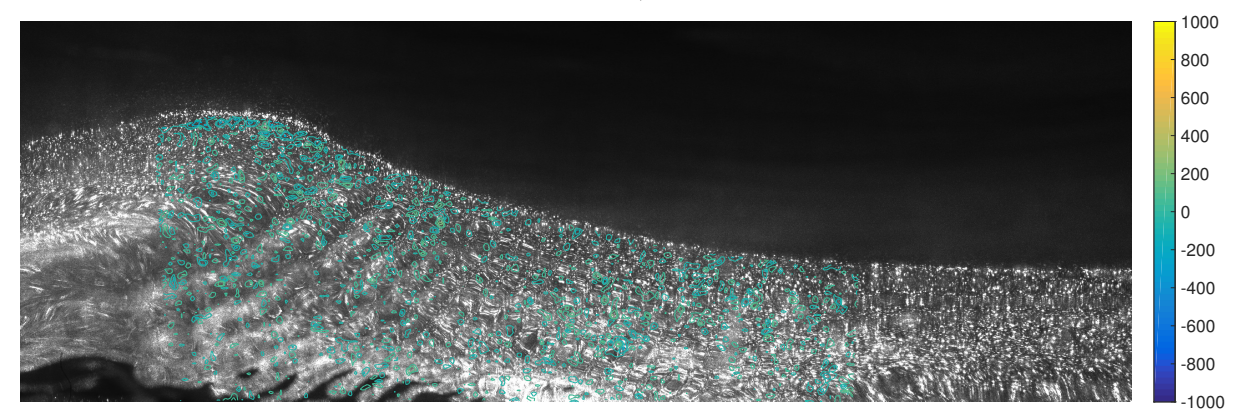

d)

FIG. 8: Example images. $U_{s l}=0.1, U_{s g}=2.40$. a) Interface seen from LFV camera. b) Vector plot seen in a frame of reference moving with the wave speed. c) Vorticity field below the crest (colorbar in $\left[s^{-1}\right]$ ). d) PIV interface detection camera with liquid phase vorticity field superimposed (colorbar in $\left[s^{-1}\right]$ ). Note that only a section of the liquid phase PIV images (b and c) are shown. In figure $b$ half of the vectors are shown. Flow from left to right. 


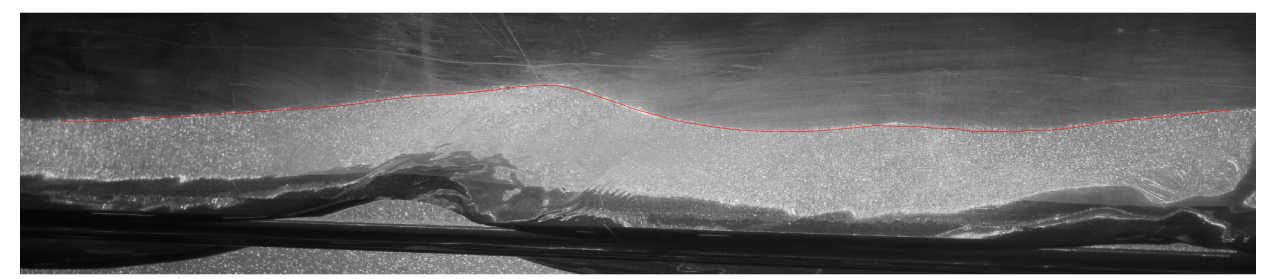

a)

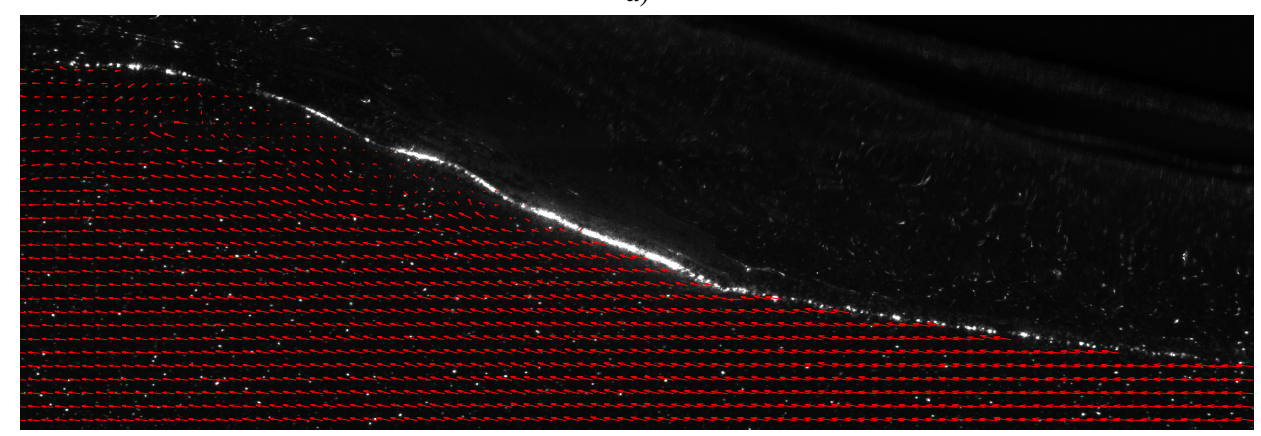

b)

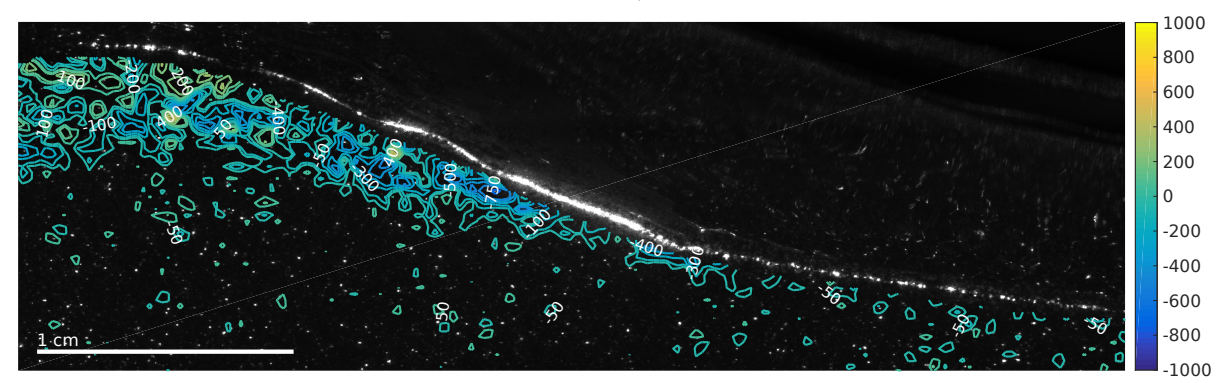

c)

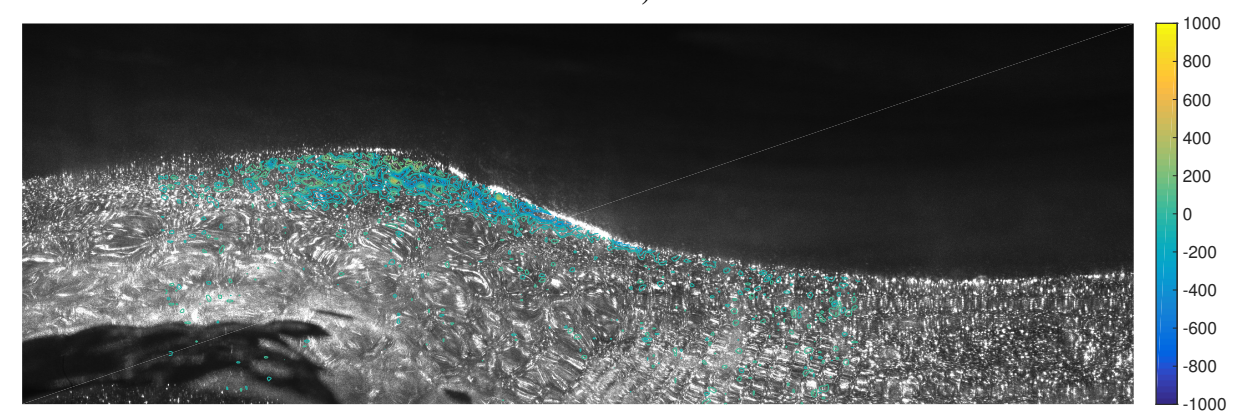

d)

FIG. 9: Same as figure 8. 


\section{B. ROI statistics}

Based on the instantaneous velocity fields obtained, a region of interest (ROI) was defined, and the flow properties in this region were monitored to detect the signature of breaking waves.

For all the waves where clear indications of active wave breaking was observed, high levels of vorticity was observed on the leeward side of the crest, associated with the spilling region of the wave. The full extent of this high vorticity region varied considerably, as different stages of the wave breaking process was detected. However, in the region from $20^{\circ}<\theta<80^{\circ}$, from the interface and down to approximately $3 \mathrm{~mm}$ below the interface, high levels of vorticity was always present for the waves associated with wave breaking. This region was selected as the ROI (indicated in figure 10). All PIV velocity fields containing the ROI were analyzed (number of PIV velocity fields including the ROI presented in table II), and statistics from the ROI analyzed. The main focus is on evaluating the rms vorticity (denoted $\omega_{\text {rms }}$ ) and the maximum horizontal velocity in the ROI.

In figure 11 a) scatter plot of the maximum horizontal velocity divided by the wave velocity $\left(u_{\max } / c\right)$ versus $\omega_{\text {rms }}$ in the $\mathrm{ROI}$ is presented for all waves analyzed. The wave speed $c$ is evaluated for each wave individually by a cross correlation of the upstream pressure probes, used to trigger the PIV system (ref. "Appendix A: Trigging of PIV system"). As the figure contains many overlapping data points, lines are included to indicate the maximum extent of the scatter points for each experimental case. In figure $11 \mathrm{~b}) \omega_{r m s}$ is binned with a bin size of 10 , and the probability density function (PDF) of $\omega_{\text {rms }}$ within the ROI is presented. Figure 11 a) shows that the relationship between $\omega_{\text {rms }}$ and $u_{\max } / c$ can be divided into two regimes, and the transition between these occur at $u_{\text {max }} / c \approx 1$. Above this limit the slope of $\omega_{r \operatorname{ms}}\left(u_{\max } / c\right)$ is seen to increase significantly. This result is to be expected, as wave breaking is characterized by fluid elements near the wave crest overtaking the general waveform resulting in increased vorticity beneath the crest.

In figure $11 \mathrm{~b}$ ) it can be observed that for the two lowest flow rate cases, $\omega_{r m s}$ is in the range of 20 to 60 for all waves, except for one observation of the $U_{s g} 1.70 \mathrm{~m} / \mathrm{s}$ case. For all experimental cases a distinct peak is observed in the PDF at $\omega_{r m s}$ of $30-50 s^{-1}$. This peak is associated with non-breaking waves, and the vorticity levels observed can be viewed as a background vorticity in the flow. For the two highest flow rates, a secondary, although much wider, peak is observed in the PDF at $\omega_{\text {rms }}$ between 100 and $250 \mathrm{~s}^{-1}$. These high levels of $\omega_{r m s}$ are associated with wave breaking events. As the waves are captured by the PIV system at different stages of the wave breaking process, and as the waves are likely to break with different intensity, it is to be expected that this secondary peak is wider than the peak associated with nonbreaking waves.

For the $U_{s g} 1.80 \mathrm{~m} / \mathrm{s}$ case, values of $\omega_{r m s}$ significantly higher than the peak associated with the non-breaking peak are evident, although no secondary peak in the PDF is observed, indicating that the wave breaking events are rarer and less energetic than for the $U_{s g} 2.10$ and $2.40 \mathrm{~m} / \mathrm{s}$ cases.

In figure 12, scatter plots of $\omega_{r m s}$ against two different steepness measures are presented. $S_{\max }$ is the maximum slope along the leeward side of the wave $(\max (|d \eta / d x|))$, while $a k=\pi H / \lambda$, where $H$ and $\lambda$ is the wave height and length, evaluated from the LFV camera. Note that the interface was detected for segments of approximately $1.3 \mathrm{~mm}$ along the wave profile, introducing a smoothing of the interface. Hence, the very high steepness associated with any capillary waves will not be resolved in the evaluation of $S_{\max }$. The results show that there is no clear correlation between $\omega_{\text {rms }}$ and the wave steepness. There does however seem to be a limiting steepness below which no waves are breaking. This limit is approximately at $S_{\max } \approx 0.2$ and $a k \approx 0.08$.

As our PIV system detects the waves at one instant in time, and at different stages of the breaking process, these results are to be expected. Peirson and Banner ${ }^{10}$ applied a local slope threshold of 0.5 to separate breaking and non-breaking waves. However, as discussed by Siddiqui and Loewen ${ }^{19}$ there will be a significant overlap in the PDF of the wave steepness when comparing breaking and non-breaking waves. While it is observed from figure 12 that waves with a local steepness exceeding 0.5 are likely to be in a state of breaking (associated with high levels of $\omega_{r m s}$ ), it does not serve as an accurate criterion for detecting breaking waves in the present work.

While the current dataset is not suited to detect the onset of wave breaking (as this would require time-resolved measurements of individual waves going from a non-breaking to breaking state), the maximum steepness observed may be interpreted as a limiting steepness for the waves in the system. The maximum $a k$ observed is $\approx 0.3$. This is a typical steepness where wave-breaking in the ocean is assessed to occur ${ }^{12}$.

\section{Breaking detection method}

Based on the observations of the ROI statistics, a criterion for distinguishing breaking and non-breaking waves is introduced. Figure 13 illustrates the fraction of waves assessed to be breaking using different thresholds for $\omega_{\text {rms }}$, and by applying $u_{\max }>c$ as a criterion. This illustrates that the fraction of breaking waves is sensitive to the threshold applied, but that for the range of thresholds considered to be reasonable, the onset of wave breaking occurs at the same flow rate case $\left(U_{s g}\right.$ $=1.8 \mathrm{~m} / \mathrm{s}$ ), and the trends in the observed breaking frequency do not vary considerably depending on the threshold applied.

In the rest of this analysis, waves with $\omega_{\text {rms }}<60$ are assessed to be non-breaking, while waves with $\omega_{r m s}>100$ are assessed to be breaking. Referring to figure 11 a) this threshold ensures that virtually all waves assessed to be breaking has $u_{\max } / c>1$. Employing $u_{\max } / c>1$ as a criterion directly is not assessed to be robust, as this is only dependent on one vector in the ROI, and because of uncertainties related to determining the instantaneous wave speed. It should be noted that these thresholds are not general, and will depend on the PIV resolution of the measurements (as higher resolution implies that more of the shear is resolved).

The total number of PIV velocity fields, waves with ROI 


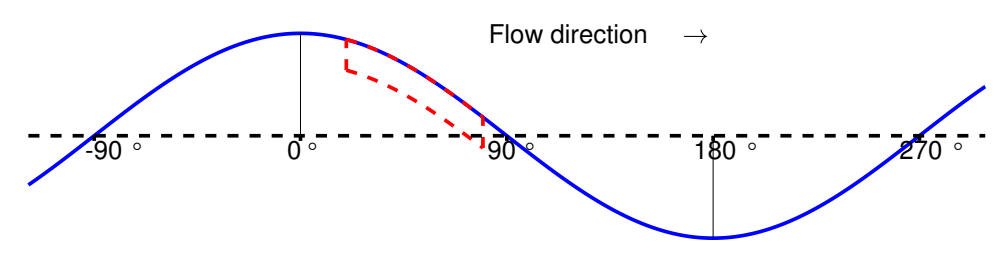

FIG. 10: Illustration of the region of interest. Blue line represents water surface. Red dotted line contains the ROI.

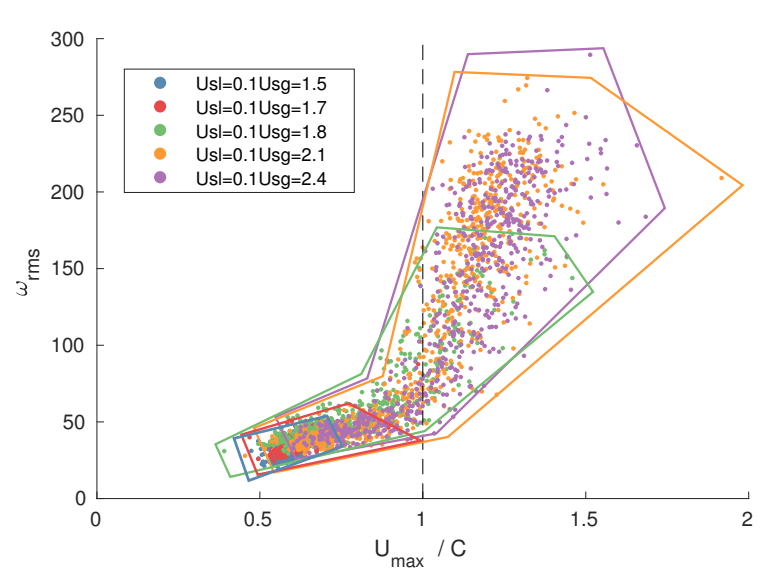

a)

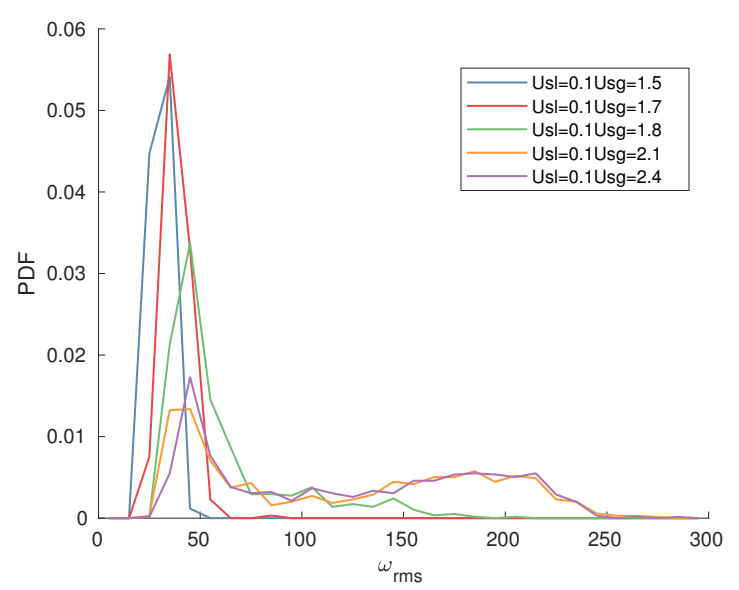

b)

FIG. 11: a) Scatter plot of maximum horizontal velocity divided by wave speed vs. rms vorticity [ $\left.s^{-1}\right]$ observed in ROI. Lines corresponding to color of each experimental case illustrates the maximum extent of the scatter points for each case. b) Probability distribution of rms vorticity $\left[s^{-1}\right]$ within the ROI.

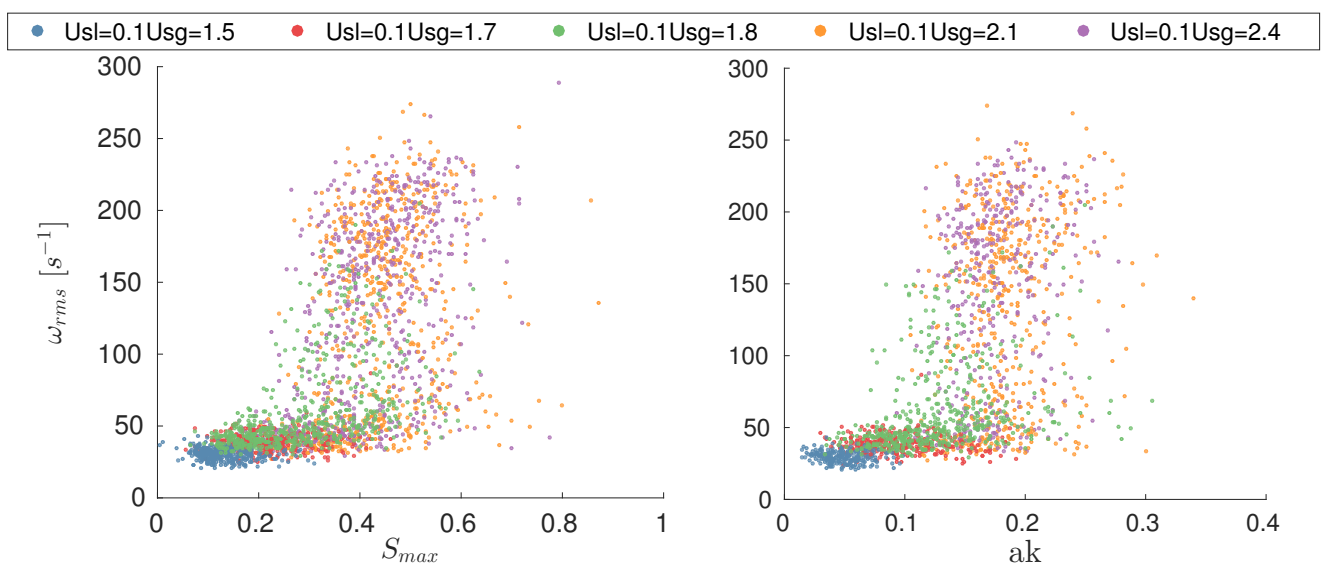

FIG. 12: Scatter plot of $\omega_{r m s}$ vs. maximum wave slope $S_{\max }$ and the wave steepness $a k$.

within the PIV FOV and waves assessed to be breaking and non-breaking are presented in table II. Waves with $60<$ $\omega_{\text {rms }}<100$ are not classified as either breaking or nonbreaking. In table II they are classified as "intermediate". These might be waves at a very early/late stage of the breaking process, or it could be non-breaking waves passing through the turbulent wake of an upstream breaking wave. Inspection of the velocity fields related to these cases illustrated that it was sometimes difficult to assess whether the waves were breaking or not, and that setting a single threshold for separating breaking from non-breaking waves resulted in cases where non-breaking waves were assessed to be breaking and vice versa.

Siddiqui and Loewen ${ }^{19}$ also used a criterion based on the vorticity in the crest region to differentiate between breaking and non-breaking waves in an open channel system. They 


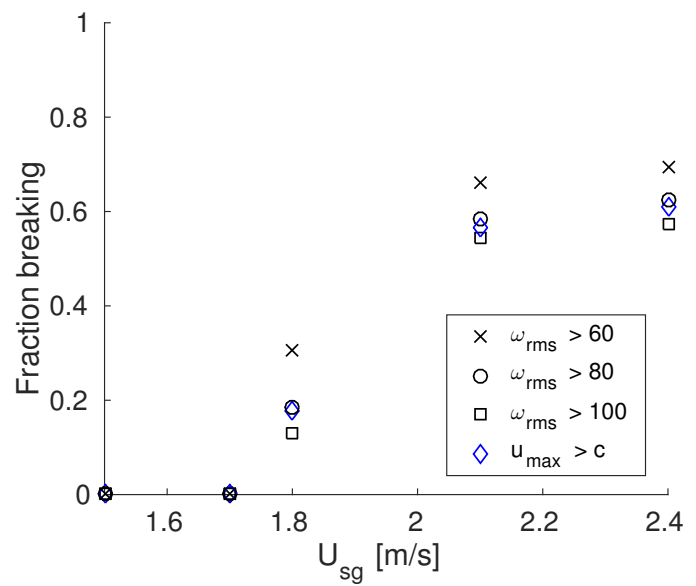

FIG. 13: Fraction of waves that are considered breaking at different $\omega_{r m s}$-threshold values, and using $u_{\max }>c$ as criterion for detecting wave breaking.

considered waves where the variance of the vorticity in the ROI (note that a different ROI was applied) was greater than $70 s^{-2}$ to be microscale breaking. The present study considers a different physical system than what was investigated by Siddiqui and Loewen ${ }^{19}$, and the geometrical properties of the waves vary somewhat between the two studies. Differences in the observed vorticity levels is for this reason to be expected. However, it is assessed that the primary reason for the significantly higher vorticity levels observed in the present work is the enhanced PIV resolution (resolution is a factor 8 times higher compared with Siddiqui and Loewen ${ }^{19}$ ). The vorticity levels observed in this work resemble the results from Peirson and Banner ${ }^{10}$ and Duncan ${ }^{8}$, where the PIV resolution is similar to our analysis. The more focused ROI applied in the present work also means that a higher value of the vorticity threshold is suitable compared with the analysis performed by Siddiqui and Loewen ${ }^{19}$.

\section{Characteristics of breaking and non-breaking waves}

Using the $\omega_{r m s}$-threshold for detecting breaking and nonbreaking waves described in section IV B 1, statistics of breaking and non-breaking waves are extracted and compared. As there are five experimental cases, for three of which we observe both non-breaking and breaking waves, a total of eight averaging cases are analyzed.

\section{Average properties of breaking and non-breaking waves}

In table III the mean of the maximum wave steepness $\left(\overline{S_{\max }}\right), \overline{a k}$, ROI steepness $\left(\overline{S_{R O I}}\right.$, evaluated as $\left.|\Delta y / \Delta x|\right)$ in the ROI) and average crest height $\left(\overline{\eta_{c}}\right)$ for microbreaking and nonmicrobreaking waves are compared. Note that for $U_{s g}=1.50$ and $1.70 \mathrm{~m} / \mathrm{s}$, no breaking waves were detected. It is observed that on average, the breaking waves are steeper than the non-breaking waves, evaluating either of the three steepness measures. These results are similar to the results reported by Siddiqui and Loewen ${ }^{38}$, who observed that $\overline{S_{\max }}$ increased by $30-50 \%$ comparing breaking and non-breaking waves. Note that as discussed previously there is a significant overlap in the steepness observed for breaking and non-breaking waves, hence the steepness in itself can not be used as a criterion for detecting breaking waves.

While Siddiqui and Loewen ${ }^{38}$ observed that microscale breaking waves on average had a higher crest than nonbreaking waves (25 - $100 \%$ higher), results for the two highest flow rates imply that there is very little difference in the crest height of breaking and non-breaking waves observed in the present work. The reason for this discrepancy is assessed to be the triggering system applied. While Siddiqui and Loewen ${ }^{38}$ evaluated all waves passing the PIV section as either breaking or non-breaking, the triggering system introduces a bias towards detecting larger amplitude waves. Including a large fraction of small amplitude, non-breaking waves into the dataset would result in the average crest height of non-breaking waves to be reduced, and our results would likely be more in accord with the results obtained by Siddiqui and Loewen ${ }^{38}$.

\section{Phase-averaged properties of breaking and non-breaking waves}

The conditional phase-averaging procedure described in section III C was applied to all five experimental cases, separating breaking and non-breaking waves. In figure 14, phaseaveraged properties for the $U_{s g}=2.10 \mathrm{~m} / \mathrm{s}$ case is presented. These are representative for the results obtained also at $U_{s g}=$ 1.80 and $2.40 \mathrm{~m} / \mathrm{s}$. The subfigures on the left illustrate phaseaveraged properties beneath non-breaking waves, while the subfigures on the right illustrate properties of the breaking waves. The velocity fields are plotted in a region ensuring that at least 200 data points are used in the averaging of any variable in the $(\theta, \zeta)$ coordinate system. When analyzing the phase-averaged results, it should be kept in mind that the averaging is performed over a relatively broad-banded wave spectrum, hence significant variability is expected also within each averaging case. However, the phase-averaged velocity fields are assessed to provide valuable information on the characteristic features of the waves, and how these evolve from nonbreaking to active breaking.

Several distinct features of the flow fields can be observed in figure 14. Comparing figure $14 \mathrm{a}$ ) and b) it is observed that while the peak horizontal velocity for non-breaking waves is centered at the crest, this peak is shifted to the leeward side of the breaking waves, indicating a spilling region. This spilling region is also observed clearly in the $\langle V\rangle$ plots presented in figure $14 \mathrm{c}$ ) and d).

In figure $14 \mathrm{e})$ - h) the phase-averaged $\omega_{z}$ - and $\varepsilon$-fields reveal that while the vorticity is observed to be fairly constant over the wave form in the non-breaking case, significantly higher values (approximately one order of magnitude) are observed in the spilling region for the breaking waves. This is consistent with the instantaneous velocity fields analyzed in 
TABLE II: Overview of waves analyzed by PIV, and fraction of breaking and non-breaking waves.

\begin{tabular}{|l|c|c|c|c|c|}
\hline$U_{s g}$ & 1.50 & 1.70 & 1.80 & 2.10 & 2.40 \\
\hline Number of PIV velocity fields & 500 & 500 & 1000 & 1000 & 1000 \\
\hline Waves with ROI identified & 345 & 306 & 582 & 696 & 654 \\
\hline Number (percentage) breaking & $0(0)$ & $0(0)$ & $75(12.8)$ & $379(54.5)$ & $374(57.2)$ \\
\hline Number (percentage) intermediate & $0(0)$ & $1(0.3)$ & $101(17.3)$ & $81(11.6)$ & $80(12.2)$ \\
\hline Number (percentage) non-breaking & $345(100)$ & $305(99.7)$ & $406(69.8)$ & $236(33.9)$ & $200(30.6)$ \\
\hline
\end{tabular}

TABLE III: Average properties of breaking and non-breaking waves. $\eta_{c}$ is the crest height. Overbar indicates average properties.

\begin{tabular}{|c|c|c|c|c|c|c|c|c|}
\hline \multirow{2}{*}{$U_{s g}$} & \multicolumn{2}{|c|}{$\overline{S_{\max }}[-]$} & \multicolumn{2}{c|}{$\overline{a k}[-]$} & \multicolumn{2}{c|}{$\overline{S_{R O I}}[-]$} & \multicolumn{2}{c|}{$\overline{\eta_{c}}[\mathrm{~mm}]$} \\
\cline { 2 - 9 } & $\begin{array}{c}\text { non- } \\
\text { breaking }\end{array}$ & breaking & $\begin{array}{c}\text { non- } \\
\text { breaking }\end{array}$ & breaking & $\begin{array}{c}\text { non- } \\
\text { breaking }\end{array}$ & breaking & $\begin{array}{c}\text { non- } \\
\text { breaking }\end{array}$ & breaking \\
\hline 1.50 & 0.13 & NA & 0.049 & NA & 0.04 & NA & 0.8 & NA \\
\hline 1.70 & 0.22 & NA & 0.096 & NA & 0.10 & NA & 2.1 & NA \\
\hline 1.80 & 0.25 & 0.38 & 0.111 & 0.142 & 0.15 & 0.26 & 3.9 & 4.8 \\
\hline 2.10 & 0.35 & 0.46 & 0.164 & 0.180 & 0.25 & 0.32 & 7.2 & 7.0 \\
\hline 2.40 & 0.33 & 0.46 & 0.157 & 0.170 & 0.22 & 0.31 & 7.3 & 7.2 \\
\hline
\end{tabular}

section IV A. Also $\langle\varepsilon\rangle$ is observed to have peak values one order of magnitude higher for the breaking than non-breaking waves. It can be noted that the maximum dissipation rate is observed on the leeward side of the wave, in the highly turbulent spilling region. Siddiqui and Loewen ${ }^{20}$ also calculated the phase distribution of $\varepsilon$, and found that the peak values of $\varepsilon$ were located directly below the wave crest. As the spilling region is very thin (typically less than $2 \mathrm{~mm}$ ), it seems likely that this was not captured by the measurements of Siddiqui and Loewen ${ }^{20}$, as their PIV resolution was approximately 2 $\mathrm{mm}$. The results presented in figure 14 are assessed to be qualitatively reasonable, as we expect high levels of energy dissipation in the spilling region. However, as discussed in section III D, we do not expect that the PIV resolution is sufficient to accurately estimate $\varepsilon$ in this region, as the very high dissipation rates observed imply a Kolmogorov length scale significantly smaller than the PIV resolution.

In figure 15 the phase-averaged interface elevation $\eta$ for breaking and non-breaking waves is presented in figure a). In figure $15 \mathrm{~b}$ ) and c), $\left\langle\omega_{z}\right\rangle$ and $\langle\varepsilon\rangle$ are depth-averaged over the top $2 \mathrm{~mm}$ of the wave (typical depth of the highly turbulent spilling region), and presented as a function of wave phase.

The phase-averaged interface elevation is calculated by the interface detected by the LFV camera, hence for all cases a reliable average is obtained for all wave phases. As $\left\langle\omega_{z}\right\rangle$ and $\langle\varepsilon\rangle$ is calculated by the PIV velocity fields, information on these parameters far from the ROI $\left(20^{\circ}\right.$ to $\left.80^{\circ}\right)$ are not available. A cut off in the data is made so that phase-averaged values with fewer than 50 data points are omitted from the results presented in figure 15. All breaking and non-breaking waves (numbers listed in table II) will contain information of the wave phase from $20^{\circ}$ to $80^{\circ}$, while the probability of detecting a wave phase away from the ROI decreases with distance from the ROI.

Consistent with the results for $\left\langle\eta_{c}\right\rangle$ presented in table III, it can be observed that there is little to differentiate the observed crest heights of breaking and non-breaking waves of the two highest flow rates. However, the troughs of the breaking waves are significantly shallower. This is consistent with the results presented in table III, where it was observed that the average wave steepness $\overline{a k}$ is higher for the breaking than for the non-breaking waves, indicating that the breaking waves (on average) has a higher degree of non-linearity.

The results for the depth-averaged $\left\langle\omega_{z}\right\rangle$ and $\langle\varepsilon\rangle$ follow the same trends observed in figure $14 \mathrm{e})$-h). Significantly higher levels of vorticity and turbulent dissipation is observed in the spilling region of breaking waves compared with the nonbreaking waves.

In figure $16 a$ ) and b) the phase-averaged horizontal velocity and turbulent dissipation rate below the crest (at $\theta=0^{\circ}$ ) is presented for all averaging cases. It is observed that for the $U_{s g} 2.10$ and $2.40 \mathrm{~m} / \mathrm{s}$ cases, the phase-averaged horizontal velocity for breaking and non-breaking waves is very similar below a depth of approximately $6 \mathrm{~mm}$ from the interface. Closer to the interface the horizontal velocity profiles of the breaking waves are observed to increase compared with the non-breaking waves.

The results for $\langle\varepsilon\rangle$ beneath the crest of microscale breaking waves shows that the turbulent dissipation rate is approximately $1.5 * 10^{-2} \mathrm{~m}^{2} / \mathrm{s}^{3}$ close to the water surface. This is an order of magnitude higher than the results reported by Siddiqui and Loewen ${ }^{38}$. While there are differences in the physical system (comparing an open wind-wave tank and a closed two-phase pipe flow geometry) the higher PIV resolution in the present work is assessed to be an important reason for the enhanced turbulent dissipation rate calculated. Lee et al. ${ }^{42}$ reported values of $\varepsilon$ below the crest of mechanically generated microscale breaking waves, and found values approaching $1 * 10^{-2} \mathrm{~m}^{2} / \mathrm{s}^{3}$ near the interface, in line with the dissipation rates observed here. It should be noted that the calculation of $\varepsilon$ is sensitive to errors in the PIV. These are (in absolute value) assessed to be relatively constant within the PIV FOV. As we see a clear evolution of $\varepsilon$ with depth, and a significant increase in the turbulent dissipation rate for breaking waves, we assess that the turbulent dissipation rate near the interface (for breaking and non-breaking waves) is not dominated by noise from the PIV. Care should however be taken when evaluating the lowest dissipation rates observed, as these may be 


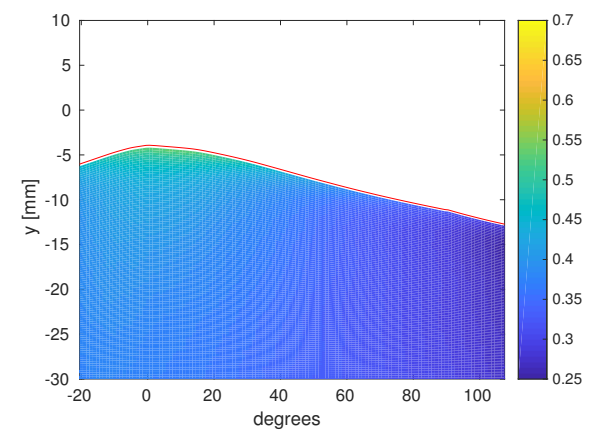

a) $\langle U\rangle[\mathrm{m} / \mathrm{s}]$, non-breaking

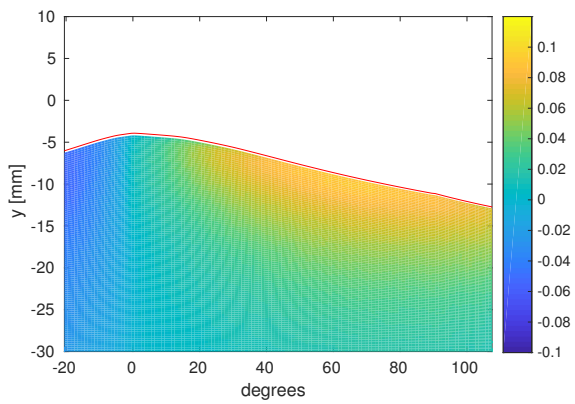

c) $\langle V\rangle[\mathrm{m} / \mathrm{s}]$, non-breaking

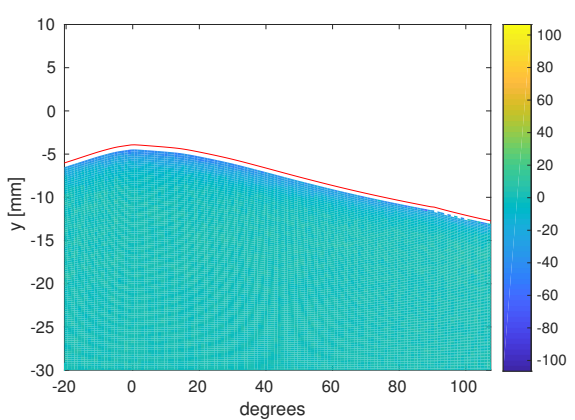

e) $\left\langle\omega_{z}\right\rangle\left[s^{-1}\right]$, non-breaking

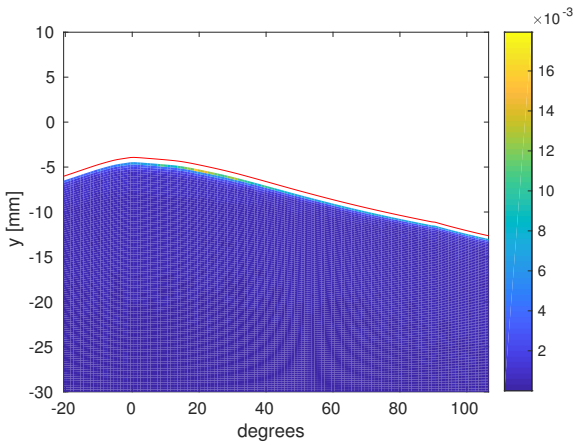

g) $\langle\varepsilon\rangle\left[\mathrm{m}^{2} / \mathrm{s}^{3}\right]$, non-breaking

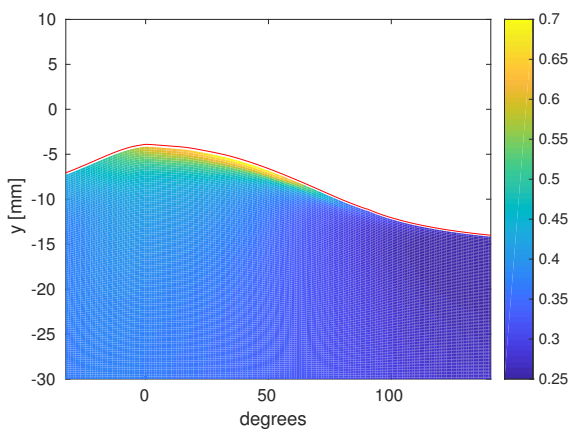

b) $\langle U\rangle[\mathrm{m} / \mathrm{s}]$, breaking

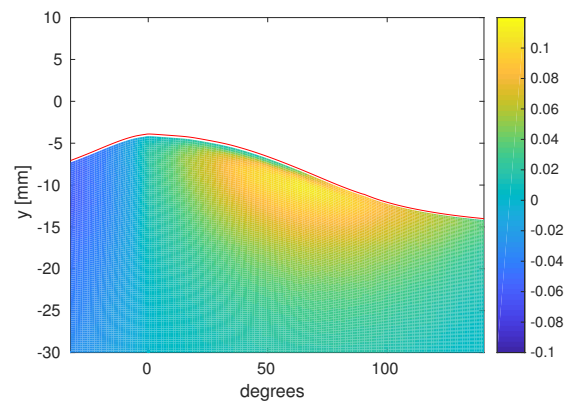

d) $\langle V\rangle[\mathrm{m} / \mathrm{s}]$, breaking

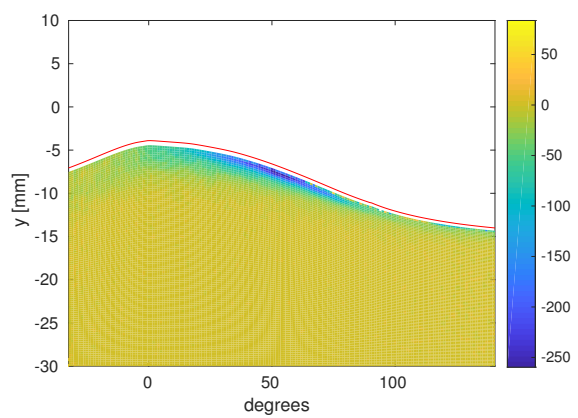

f) $\left\langle\omega_{z}\right\rangle\left[s^{-1}\right]$, breaking

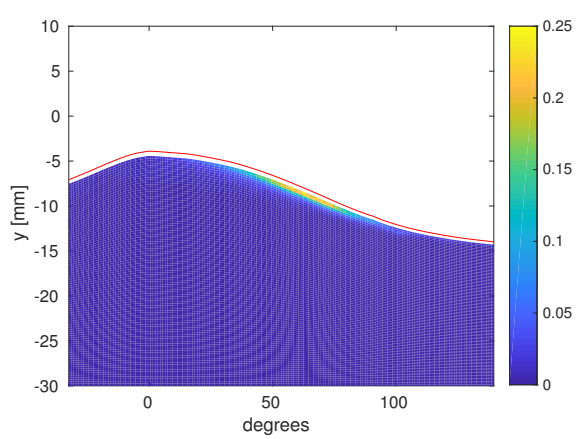

h) $\langle\varepsilon\rangle\left[m^{2} / s^{3}\right]$, breaking

FIG. 14: Comparison of phase-averaged fields for non-breaking (left side) and breaking (right side) waves observed in the $U_{s g}$ $2.10 \mathrm{~m} / \mathrm{s}$ case.

significantly influenced by noise in the PIV measurements.

The black dashed lines in figure $16 \mathrm{~b}$ ) indicate that for the non-breaking cases, and below the depth where the increased dissipation rate associated with wave breaking is observed, $\varepsilon \propto \xi^{-0.7}$, where $\xi$ is the distance from the interface. In the region directly affected by wave breaking the dissipation rate is found to scale approximately as $\varepsilon \propto \xi^{-2}$. Based on analysis of field experiments conducted in Lake Ontario, Terray et al. ${ }^{43}$ proposed a three-layer scaling of the dissipation rate below breaking waves. Terray et al. ${ }^{43}$ proposed that breaking 


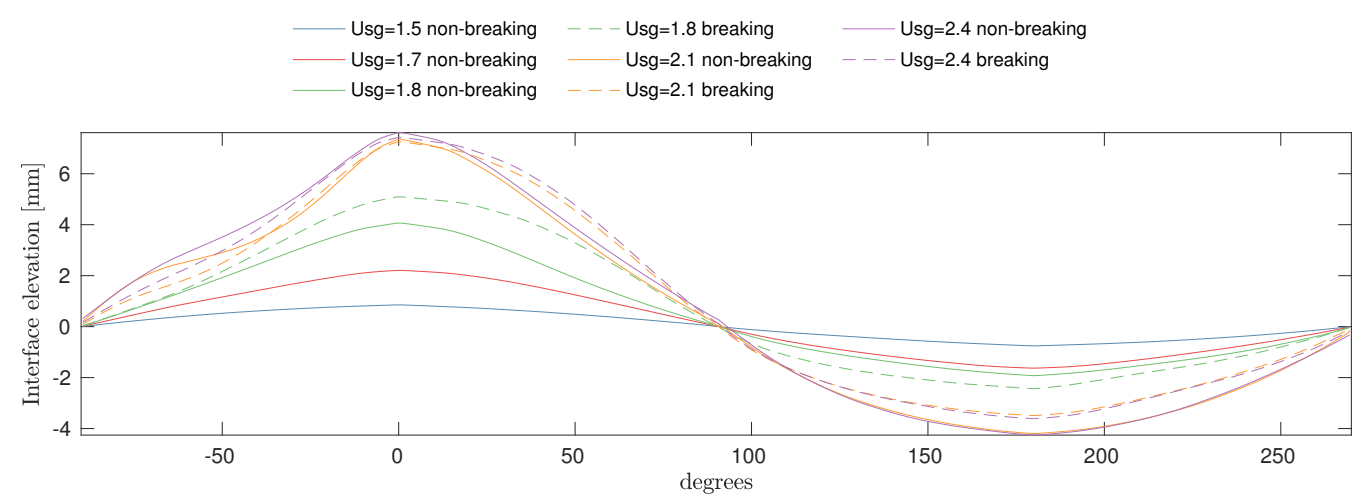

a) $\langle\eta\rangle$

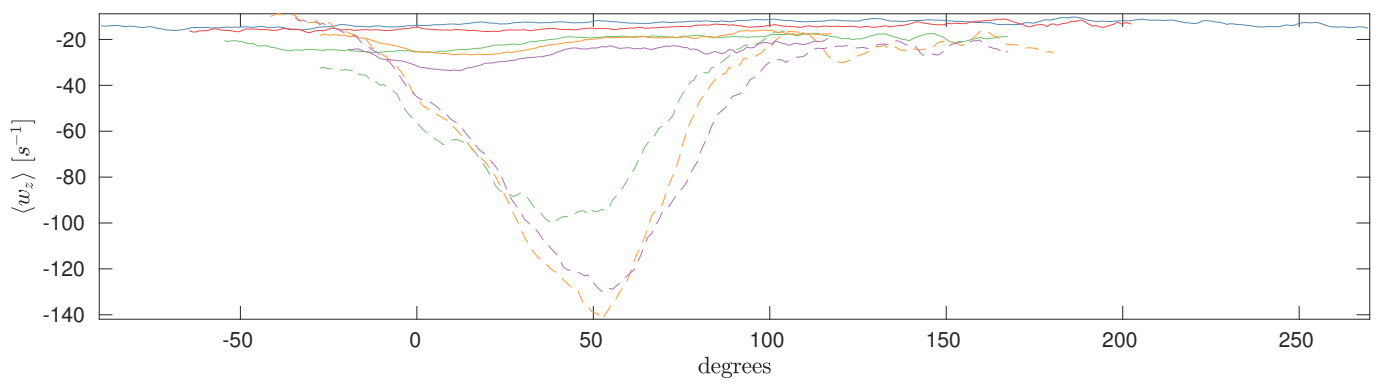

b) $\left\langle\omega_{z}\right\rangle$ near interface

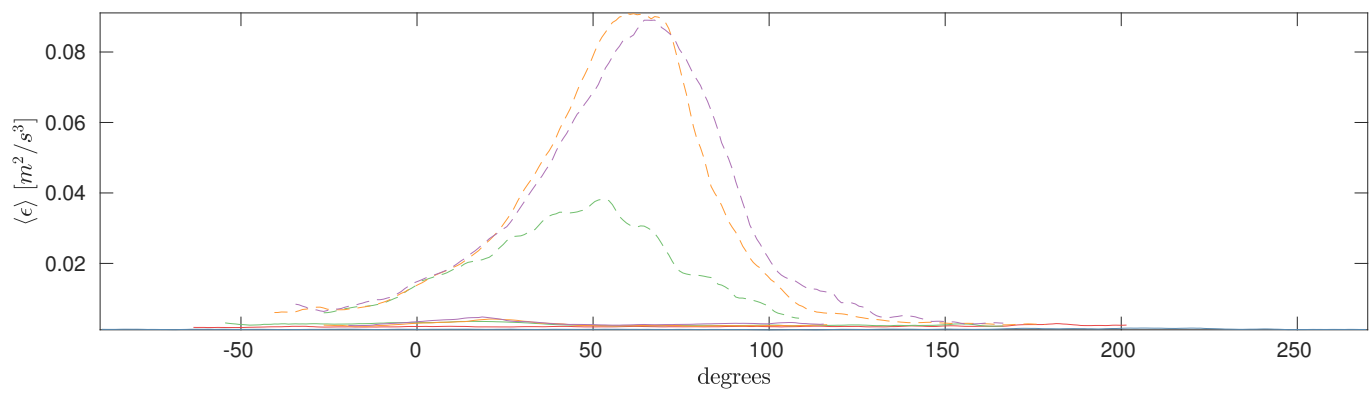

c) $\langle\varepsilon\rangle$ near interface

FIG. 15: a) Mean interface elevation as function of wave phase. b) and c) Phase-averaged properties averaged within the top 2 $\mathrm{mm}$ of the interface.

directly injects energy to a depth $z_{b}$, and the dissipation rate was assumed to be constant between the surface and $z_{b}$. Below this upper layer was an intermediate layer which was found to scale as $\varepsilon \propto \xi^{-2}$, similar to the behavior observed in figure $16 \mathrm{~b})$. Below the transition layer was a region which would behave as a normal wall-layer. Terray et al. ${ }^{43}$ identified the "breaking depth" as $z_{b} \approx 0.6 H_{s}$, where $H_{s}$ is the significant waveheight. The constant dissipation rate above the breaking depth is not observed in the data presented in figure $16 \mathrm{~b}$ ). However, it should be kept in mind that there are significant differences to the physical systems analyzed, and the breaking severity of microscale and larger scale breaking waves. Lee et al. ${ }^{42}$ reports $\varepsilon \propto \xi^{-2}$ beneath the crest of breaking waves, and while a constant dissipation rate layer at the scale of the significant wave height was not observed, the dissipation was found to scale as $\varepsilon \propto \xi^{-1}$ close to the surface (for depths less than $\approx 0.3$ times the wave amplitude). For the $U_{s g} 2.10$ and $2.40 \mathrm{~m} / \mathrm{s}$ cases (considering breaking waves), we also observe a change in the depth-dependence of $\varepsilon$ close to the free surface, although we do not observe the same scaling as reported by Lee et $a l .{ }^{42}$.

From figure $16 \mathrm{~b}$ ) the effect of microscale breaking is observed down to a depth of approximately $10 \mathrm{~mm}$ below the interface for the two highest gas flow rate cases. This is consistent with the results from Siddiqui and Loewen ${ }^{38}$, who found that the effect of microscale breaking waves could be observed to a depth of one significant waveheight $\left(H_{s}=4 \eta_{r m s}\right.$, is approximately $12 \mathrm{~mm}$ for these cases). To obtain a more global estimate of the effect of breaking on the turbulent dissipation rate, $\langle\varepsilon\rangle$ is depth-averaged from the interface down to $10 \mathrm{~mm}$ below the interface at the crest $\left(\theta=0^{\circ}\right)$. The results are presented in figure $16 \mathrm{c}$ ). It is observed that $\langle\varepsilon\rangle$ remains rel- 
atively constant below non-breaking waves as the gas flow rate is increased above the onset of wave breaking. However, below breaking waves the dissipation rate is observed to increase as the gas velocity is increased, indicating that the breaking events become more energetic. Comparing the depth-averaged dissipation rate of breaking and non-breaking waves it is observed that the dissipation rate is 2.5-4 times higher for the breaking cases. These results are comparable to the results presented by Siddiqui and Loewen ${ }^{20}$, who reported an increase in turbulent dissipation rate by a factor 2-3 beneath the crest of microscale breaking waves.

\section{Estimation of total breaking frequency}

The breaking frequencies presented in figure 13 and table II represent the breaking frequencies observed in the PIV data acquired by the pressure probe trigging. As shown in "Appendix A: Trigging of PIV system" the trigging system results in a bias towards detecting and acquiring data for the higher amplitude waves in the system. To estimate a total breaking frequency for all waves, the breaking probability within each $0.5 \mathrm{~mm}$ crest height bin observed by the PIV was evaluated, using the threshold of $\omega_{r m s}>100$. The number of observations within each crest height bin observed by the wave probes was then used to estimate the total breaking frequency for each experimental case. Results are presented in figure 17.

For the two highest gas velocity cases, there are no observed wave crests with a surface elevation below $3.5 \mathrm{~mm}$ in the PIV dataset. Here the breaking probability is assumed to be zero. This is assessed to be a reasonable estimate as for the two height bins from 3.5 to $4.5 \mathrm{~mm}$, a marked reduction in the breaking probability (approximately $50 \%$ reduction) was observed compared with the higher amplitude waves for both cases. Extrapolating these results, and also considering the fact that no cases of wave breaking was observed for crest heights below $2.5 \mathrm{~mm}$ in the $U_{s g} 1.80 \mathrm{~m} / \mathrm{s}$ case, it seems reasonable that only a small fraction of the waves with a crest height below $3.5 \mathrm{~mm}$ in the $U_{s g}=2.10$ and $2.40 \mathrm{~m} / \mathrm{s}$ cases will be breaking.

It should be noted that there are other biases which may impact on the total breaking probability estimated. One of these is the method employed to detect breaking, which requires the region from $20^{\circ}$ to $80^{\circ}$ to be visible in the PIV FOV. This results in a bias towards detecting waves where the leeward side of the crest is steeper, and the distance from the wave crest to the zero-down crossing (at $90^{\circ}$ ) is short. As seen in section IV C 1, steeper waves are more likely to be in a state of breaking, hence this effect may result in a bias towards detecting a higher fraction of breaking waves. The threshold of $\omega_{\text {rms }}$ also impacts the total breaking frequency. This has a relatively higher impact on the $U_{s g}=1.80 \mathrm{~m} / \mathrm{s}$ case, as seen in figure 13 .

From visual inspection of the wave field and analysis of high speed video of the experiments, the breaking probabilities presented in figure 17 are assessed to be reasonable. The initial occurrence of breaking waves at $U_{s g} 1.80 \mathrm{~m} / \mathrm{s}$ coincides with the visual impression obtained from the experiments.

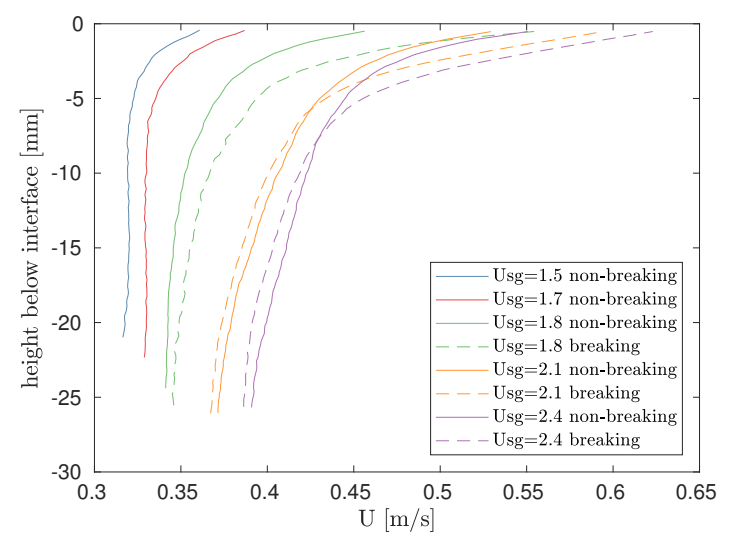

a) $\langle U\rangle$ at $\theta=0$

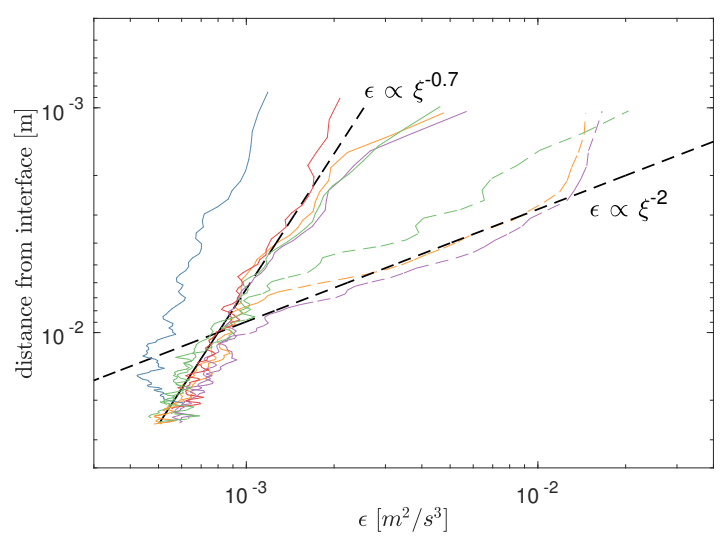

b) $\langle\varepsilon\rangle$ at $\theta=0$

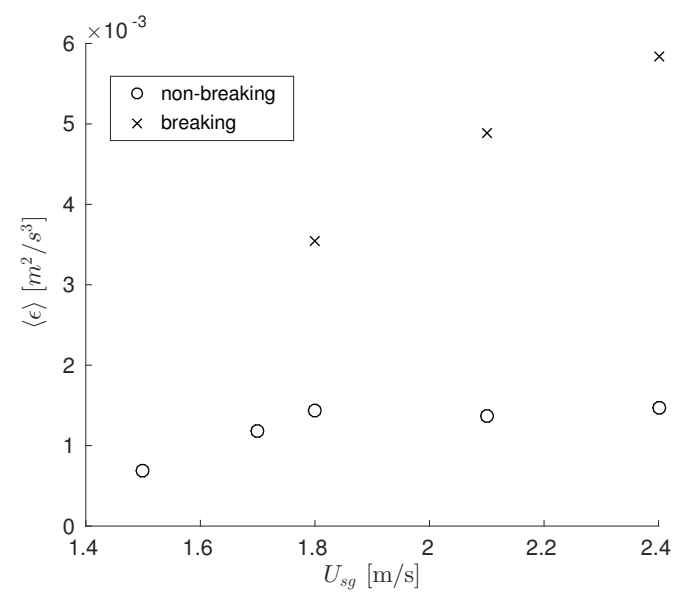

c) depth-averaged $\langle\varepsilon\rangle$ at $\theta=0$

FIG. 16: a) Phase-averaged horizontal velocity below the crest. b) Turbulent dissipation rate $\langle\varepsilon\rangle$ below crest. c) Depth-averaged $\langle\varepsilon\rangle$ below the crest, from interface to $10 \mathrm{~mm}$ below interface.

\section{CONCLUDING REMARKS}

In this paper detailed PIV measurement of waves in twophase pipe flow is performed, in order to detect where in the flow-map small-scale wave breaking is first observed, and to 


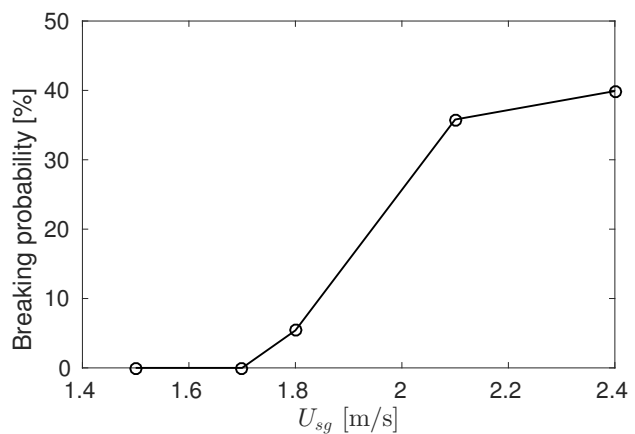

FIG. 17: Estimated total breaking probability

quantify the impact of these small-scale breaking events.

Keeping the superficial liquid velocity constant at $0.1 \mathrm{~m} / \mathrm{s}$, the first signs of wave breaking are observed at $U_{s g}=1.8 \mathrm{~m} / \mathrm{s}$. This implies that microscale wave breaking occurs before the onset of the amplitude saturation regime reported by Ayati et al. ${ }^{34}$. As the gas flow rate is increased into the amplitude saturation regime, a significant fraction of the waves are observed to be in a state of active breaking, and it is estimated that $35-40 \%$ of the waves passing through the measurement section are breaking. Although small scale breaking is also observed before the onset of the amplitude saturation regime, the results presented in this study support the suggestions by Ayati and Carneiro ${ }^{33}$ that microscale breaking is an important mechanism as the gas velocity is increased and the wave amplitude is observed to saturate.

The effect of microscale wave breaking is observed down to a depth of approximately $10 \mathrm{~mm}$ below the interface for the highest gas flow rate case $\left(U_{s g}=2.40 \mathrm{~m} / \mathrm{s}\right)$. The analysis reveals that below the crest of microscale breaking waves the dissipation rate is 2.5 to 4 times higher than below nonbreaking waves. The turbulence levels below breaking waves are observed to increase for increasing gas flow rates, indicating that the wave breaking events become more energetic as the gas flow rate is increased.

\section{ACKNOWLEDGEMENT}

The authors wish to acknowledge the strategic research initiative EarthFlows at the Faculty of Mathematics and Natural Sciences at the University of Oslo. Laboratory Head Engineer Olav Gundersen is gratefully acknowledged for the technical assistance he provided for the experimental work.

\section{APPENDIX}

\section{Appendix A: Trigging of PIV system}

Two pressure probes (Kulite XTL-190) were mounted flush to the pipe bottom, upstream of the PIV section (ref. figure 2 ). The pressure probes sampled the pressure at a frequency of $2000 \mathrm{~Hz}$. The raw data was low-pass filtered and normalized by a normalization filter based on the previous 30 seconds of measurement time. The output from the normalization filter is centered around 0 with standard deviation 1. Assuming that the measured pressure is directly related to the hydrostatic pressure above the probes (neglecting dynamic pressure and pressure variations in the air-phase above the wavy interface), and further that this is related to the interface elevation in the center of the pipe, the output from the normalization filter represents the number of standard deviations around the mean water level of the current interface elevation. Note that it is generally observed that the water level is higher towards the pipe walls (especially for the higher gas flow rate cases), which is assessed to impact on the pressure probe data, making a direct link between the measured pressure and the interface height in the center of the pipe inaccurate.

The trigger is set up to activate when a normalized pressure peak higher than some threshold value $\sigma$ is detected by the downstream pressure probe. When a valid pressure peak is detected, cross correlation is performed between the two pressure probes, evaluating the previous 1 second of data. The peak of the cross-correlation is used to estimate the wave propagation speed $c$. The distance from the pressure probes to the PIV section (approximately $28 \mathrm{~cm}$ ) and the wave propagation speed is used to evaluate the required delay time until the system is triggered. The system ensures that the section of the wave that is of interest (the leeward side of the wave crest in this study) is repeatedly present in the FOV when the PIV system is triggered, and the double images used for PIV are obtained. As seen in table II, $\approx 65 \%$ of all PIV images acquired included the ROI, and could be used in the statistical analysis of the flow field performed in this work. A minimum time delay between two trigger pulses of 5 seconds was implemented to prevent problems with the data transfer to the computer (ref. section II).

In the present study the focus was on detecting the larger amplitude waves, where wave breaking is assessed to be more probable. For this reason a threshold value of $\sigma=1.6$ was used. This ensures that the relatively higher amplitude waves are detected and evaluated. The triggering of the PIV system described in this section introduces a bias towards including waves with a higher crest height in the PIV dataset. This bias is illustrated in figure 18 , where the wave crest heights observed by the conductance wave probes (performing continuous measurements for the full experimental time period) and by the PIV system are binned in $0.5 \mathrm{~mm}$ bins, and their frequency of occurrence in the dataset is presented.

The trigging of the PIV system was applied for all experimental cases, except for the lowest gas flow rate $\left(U_{s g}=\right.$ $1.5 \mathrm{~m} / \mathrm{s})$. Here the waves were very small and the crosscorrelation from the pressure probes was dominated by noise, providing unreliable estimates for the trigging delay. For this reason the PIV system was triggered at a constant frequency of $0.2 \mathrm{~Hz}$. Note that as the waves at this flow rate were much shorter than for the higher gas flow rate cases (ref. figure 3 c), and as the wave field was observed to be more regular (ref. figure 4), the pressure probe trigging was less important for this experimental case. It can be noted from figure 18 a) that 
the the PDF of $\eta_{c}$ observed by the wave probes and PIV system closely match as no bias is introduced by the triggering. For the remaining four cases where the pressure probe trigging was applied, it is clear that the waves triggering the PIV system represents the larger waves in the system. 


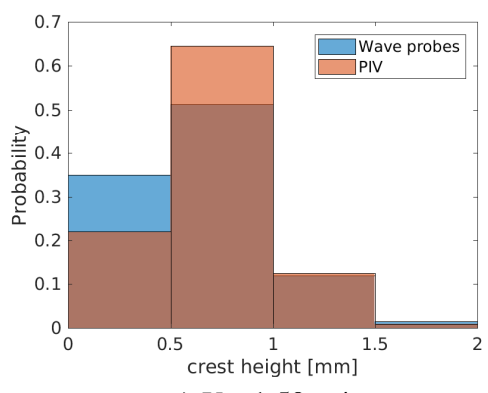

a) $U_{s g} 1.50 \mathrm{~m} / \mathrm{s}$

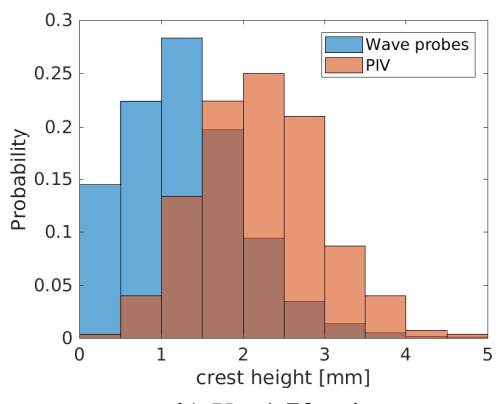

b) $U_{s g} 1.70 \mathrm{~m} / \mathrm{s}$

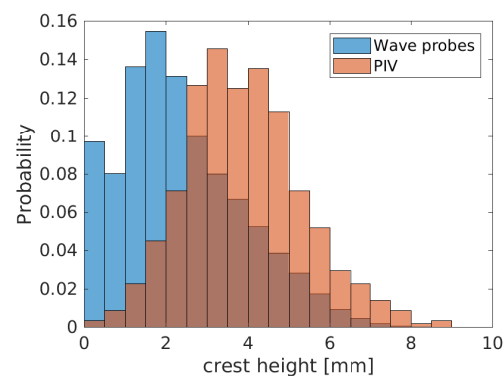

c) $U_{s g} 1.80 \mathrm{~m} / \mathrm{s}$

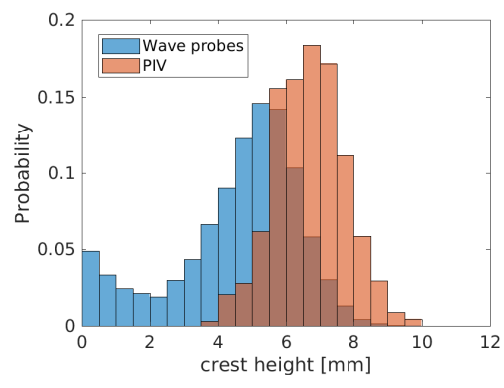

d) $U_{s g} 2.10 \mathrm{~m} / \mathrm{s}$

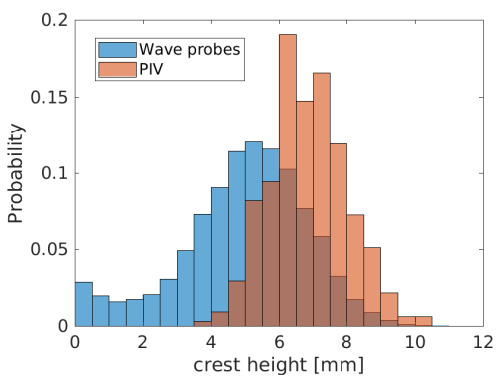

e) $U_{s g} 2.40 \mathrm{~m} / \mathrm{s}$

FIG. 18: Bar chart indicating the frequency of occurrence for crest height within $0.5 \mathrm{~mm}$ bins. Observed by wave probes (including all waves during the experimental runs) and by PIV system. 
${ }^{1}$ M. Banner and O. Phillips, "On the incipient breaking of small scale waves," Journal of Fluid Mechanics 65, 647-656 (1974).

${ }^{2}$ A. Jessup, C. Zappa, and H. Yeh, "Defining and quantifying microscale wave breaking with infrared imagery," Journal of Geophysical Research: Oceans 102, 23145-23153 (1997).

${ }^{3}$ A. Khait and L. Shemer, "On the kinematic criterion for the inception of breaking in surface gravity waves: Fully nonlinear numerical simulations and experimental verification," Physics of Fluids 30, 057103 (2018).

${ }^{4}$ M. Perlin, W. Choi, and Z. Tian, "Breaking waves in deep and intermediate waters," Annual review of fluid mechanics 45, 115-145 (2013).

${ }^{5}$ D. H. Peregrine, "Breaking waves on beaches," Annual Review of Fluid Mechanics 15, 149-178 (1983).

${ }^{6} \mathrm{~A}$. Babanin, Breaking and dissipation of ocean surface waves (Cambridge University Press, 2011).

${ }^{7}$ M. Tulin and M. Landrini, "Breaking waves in the ocean and around ships," in Twenty-Third Symposium on Naval Hydrodynamics Office of Naval Research Bassin d'Essais des Carenes National Research Council (2001).

${ }^{8}$ J. Duncan, "Spilling breakers," Annual review of fluid mechanics 33, 519547 (2001).

${ }^{9}$ L. Deike, S. Popinet, and W. Melville, "Capillary effects on wave breaking," Journal of Fluid Mechanics 769, 541-569 (2015).

${ }^{10} \mathrm{~W}$. Peirson and M. Banner, "Aqueous surface layer flows induced by microscale breaking wind waves," Journal of Fluid Mechanics 479, 1-38 (2003).

${ }^{11} \mathrm{P}$. Sutherland and W. Melville, "Field measurements and scaling of ocean surface wave-breaking statistics," Geophysical Research Letters 40, 30743079 (2013).

${ }^{12} \mathrm{P}$. Sutherland and W. Melville, "Field measurements of surface and nearsurface turbulence in the presence of breaking waves," Journal of Physical Oceanography 45, 943-965 (2015).

${ }^{13}$ G. Caulliez, "Dissipation regimes for short wind waves," Journal of Geophysical Research: Oceans 118, 672-684 (2013).

${ }^{14} \mathrm{X}$. Zhang and C. Cox, "Vortical motions under short wind waves," Winddriven air-sea interface. School of Mathematics, Uni. NSW, Sydney, Australia , 277-284 (1999).

${ }^{15}$ M. Banner, X. Barthelemy, F. Fedele, M. Allis, A. Benetazzo, F. Dias, and W. Peirson, "Linking reduced breaking crest speeds to unsteady nonlinear water wave group behavior," Physical review letters 112, 114502 (2014).

${ }^{16} \mathrm{~L}$. Shemer and D. Liberzon, "Lagrangian kinematics of steep waves up to the inception of a spilling breaker," Physics of Fluids 26, 016601 (2014).

${ }^{17} \mathrm{~K}$. Katsaros and S. Ataktürk, "Dependence of wave-breaking statistics on wind stress and wave development," in Breaking Waves (Springer, 1992) pp. 119-132.

${ }^{18}$ M. Siddiqui, M. Loewen, C. Richardson, W. Asher, and A. Jessup, "Simultaneous particle image velocimetry and infrared imagery of microscale breaking waves," Physics of Fluids 13, 1891-1903 (2001).

${ }^{19}$ M. Siddiqui and M. Loewen, "Detecting microscale breaking waves," Measurement Science and Technology 17, 771 (2006).

${ }^{20} \mathrm{~K}$. Siddiqui and M. Loewen, "Phase-averaged flow properties beneath microscale breaking waves," Boundary-Layer Meteorology 134, 499-523 (2010).

${ }^{21}$ M. Longuet-Higgins, "Capillary rollers and bores," Journal of Fluid Mechanics 240, 659-679 (1992).

${ }^{22}$ D. Tzotzi and N. Andritsos, "Interfacial shear stress in wavy stratified gasliquid flow in horizontal pipes," International Journal of Multiphase Flow $\mathbf{5 4 ,} 43$ - 54 (2013).

${ }^{23}$ M. Fernandino and T. Ytrehus, "Determination of flow sub-regimes in strat- ified air-water channel flow using LDV spectra," International journal of multiphase flow 32, 436-446 (2006).

${ }^{24}$ J. Coleman and S. Garimella, "Characterization of two-phase flow patterns in small diameter round and rectangular tubes," International Journal of Heat and Mass Transfer 42, 2869-2881 (1999).

${ }^{25} \mathrm{O}$. Strand, "An experimental investigation of stratified two-phase flow in horizontal pipes," Dr. Scient. Thesis, University of Oslo. Oslo, Norway (1993).

${ }^{26}$ N. Andritsos and T. Hanratty, "Influence of interfacial waves in stratified gas-liquid flows," AIChE journal 33, 444-454 (1987).

${ }^{27}$ D. Biberg, "A mathematical model for two-phase stratified turbulent duct flow," Multiphase Science and Technology 19 (2007).

${ }^{28}$ A. Ayati, J. Kolaas, A. Jensen, and G. Johnson, "A PIV investigation of stratified gas-liquid flow in a horizontal pipe," International Journal of Multiphase Flow 61, 129-143 (2014).

${ }^{29}$ A. Ayati, J. Kolaas, A. Jensen, and G. Johnson, "The effect of interfacial waves on the turbulence structure of stratified air/water pipe flow," International Journal of Multiphase Flow 78, 104-116 (2016).

${ }^{30} \mathrm{M}$. Birvalski, M. Tummers, R. Delfos, and R. Henkes, "PIV measurements of waves and turbulence in stratified horizontal two-phase pipe flow," International Journal of Multiphase Flow 62, 161-173 (2014).

${ }^{31}$ M. Birvalski, M. Tummers, and R. Henkes, "Measurements of gravity and gravity-capillary waves in horizontal gas-liquid pipe flow using PIV in both phases," International Journal of Multiphase Flow 87, 102 - 113 (2016).

${ }^{32}$ P. Vollestad, A. Ayati, L. Angheluta, J. LaCasce, and A. Jensen, "Experimental investigation of airflow above waves in a horizontal pipe," International Journal of Multiphase Flow 110, 37-49 (2019).

${ }^{33}$ A. Ayati and J. Carneiro, "Statistical characterization of interfacial waves in turbulent stratified gas-liquid pipe flows," International Journal of Multiphase Flow 103, 94-105 (2018).

${ }^{34}$ A. Ayati, J. Kolaas, A. Jensen, and G. Johnson, "Combined simultaneous two-phase PIV and interface elevation measurements in stratified gas/liquid pipe flow," International Journal of Multiphase Flow 74, 45-58 (2015).

${ }^{35}$ M. Raffel, C. E. Willert, F. Scarano, C. J. Kähler, S. T. Wereley, and J. Kompenhans, Particle image velocimetry: a practical guide (Springer, 2018).

${ }^{36}$ C.-T. Hsu, E. Hsu, and R. Street, "On the structure of turbulent flow over a progressive water wave: theory and experiment in a transformed, wavefollowing co-ordinate system," Journal of Fluid Mechanics 105, 87-117 (1981).

${ }^{37}$ M. Buckley and F. Veron, "Structure of the airflow above surface waves," Journal of Physical Oceanography 46, 1377-1397 (2016).

${ }^{38} \mathrm{M}$. Siddiqui and M. Loewen, "Characteristics of the wind drift layer and microscale breaking waves," Journal of Fluid Mechanics 573, 417-456 (2007).

${ }^{39}$ P. Doron, L. Bertuccioli, J. Katz, and T. Osborn, “Turbulence characteristics and dissipation estimates in the coastal ocean bottom boundary layer from PIV data," Journal of Physical Oceanography 31, 2108-2134 (2001).

${ }^{40} \mathrm{H}$. Tennekes and J. Lumley, A first course in turbulence (MIT press, 1972).

${ }^{41}$ H. Qiao and J. Duncan, "Gentle spilling breakers: crest flow-field evolution," Journal of Fluid Mechanics 439, 57-85 (2001).

${ }^{42}$ J. Lee, J. Monty, J. Elsnab, A. Toffoli, A. Babanin, and A. Alberello, "Estimation of kinetic energy dissipation from breaking waves in the wave crest region," Journal of Physical Oceanography 47, 1145-1150 (2017).

${ }^{43}$ E. Terray, M. Donelan, Y. Agrawal, W. Drennan, K. Kahma, A. Williams, P. Hwang, and S. Kitaigorodskii, "Estimates of kinetic energy dissipation under breaking waves," Journal of Physical Oceanography 26, 792-807 (1996). 